\title{
The Type 2 Diabetes Factor Methylglyoxal Mediates Axon Initial Segment Shortening and Alters Neuronal Function at the Cellular and Network Levels
}

\author{
DiDyan B. Griggs, ${ }^{1}$ Duc V. M. Nguyen, ${ }^{1}$ Leonid M. Yermakov, ${ }^{1}$ Jeneane M. Jaber, ${ }^{1}$ Jennae N. Shelby, ${ }^{1}$ \\ Josef K. Steinbrunner, ${ }^{1}$ John A. Miller, ${ }^{1}{ }^{-}$Carlos Gonzalez-Islas, ${ }^{2,3}{ }^{\circ}$ Peter Wenner, ${ }^{2}$ and \\ (- Keiichiro Susuki ${ }^{1}$
}

https://doi.org/10.1523/ENEURO.0201-21.2021

${ }^{1}$ Department of Neuroscience, Cell Biology, and Physiology, Boonshoft School of Medicine, Wright State University, Dayton, $\mathrm{OH} 45435,{ }^{2}$ Physiology Department, Emory University, School of Medicine, Atlanta, GA 30322, and ${ }^{3}$ Doctorado en Ciencias Biológicas, Universidad Autónoma de Tlaxcala, Tlax 90070, México

\begin{abstract}
Recent evidence suggests that alteration of axon initial segment (AIS) geometry (i.e., length or location along the axon) contributes to CNS dysfunction in neurological diseases. For example, AIS length is shorter in the prefrontal cortex of type 2 diabetic mice with cognitive impairment. To determine the key type 2 diabetes-related factor that produces AIS shortening we modified levels of insulin, glucose, or the reactive glucose metabolite methylglyoxal in cultures of dissociated cortices from male and female mice and quantified AIS geometry using immunofluorescent imaging of the AIS proteins AnkyrinG and $\beta$ IV spectrin. Neither insulin nor glucose modification altered AIS length. Exposure to 100 but not 1 or $10 \mu \mathrm{m}$ methylglyoxal for $24 \mathrm{~h}$ resulted in accumulation of the methylglyoxal-derived advanced glycation end-product hydroimidazolone and produced reversible AIS shortening without cell death. Methylglyoxal-evoked AIS shortening occurred in both excitatory and putative inhibitory neuron populations and in the presence of tetrodotoxin (TTX). In single-cell recordings resting membrane potential was depolarized at $0.5-3 \mathrm{~h}$ and returned to normal at $24 \mathrm{~h}$. In multielectrode array (MEA) recordings methylglyoxal produced an immediate $\sim 300 \%$ increase in spiking and bursting rates that returned to normal within $2 \mathrm{~min}$, followed by a $\sim 20 \%$ reduction of network activity at $0.5-3 \mathrm{~h}$ and restoration of activity to baseline levels at $24 \mathrm{~h}$. AIS length was unchanged at $0.5-3 \mathrm{~h}$ despite the presence of depolarization and network activity reduction. Nevertheless, these results suggest that methylglyoxal could be a key mediator of AIS shortening and disruptor of neuronal function during type 2 diabetes.
\end{abstract}

Key words: axon initial segment; depolarization; methylglyoxal; multielectrode array; network activity; type 2 diabetes

\section{Significance Statement}

Small changes in the structure of the axon initial segment (AIS) affect neuronal function and may be a key mediator of neurological complications in various disease states. However, the specific disease factors that mediate structural changes at the AIS are relatively unknown. This is the first study to show that increase of methylglyoxal is sufficient to reduce AIS length and modulate neuronal function at the cellular and network levels. Methylglyoxal is a disease factor implicated in a wide variety of conditions including type 2 diabetes, Alzheimer's disease, and aging. Thus, these findings could significantly impact the understanding of neurological complications in several disease states and are of broad pathophysiological relevance. 


\section{Introduction}

The axon initial segment (AIS) is a specialized excitable domain within neurons that maintains neuronal polarity and regulates action potential generation (Rasband, 2010; Bender and Trussell, 2012). Voltage-gated sodium channels (Nav) are anchored at the AIS by sub-membranous cytoskeletal (e.g., $\beta$ IV spectrin) and scaffolding (e.g., AnkyrinG) proteins (Nelson and Jenkins, 2017). Alteration of AIS constituents, such as mutations in ion channels or loss of protein complexes, is emerging as a key pathophysiology in a wide variety of neurological conditions in humans (for review, see Buffington and Rasband, 2011; Huang and Rasband, 2018). In the brains of animals, subtle changes in AIS geometry (i.e., length or location along the axon) are reported in models of type 2 diabetes (Yermakov et al., 2018), Alzheimer's disease (Marin et al., 2016), aging (Atapour and Rosa, 2017; Ding et al., 2018), stroke (Hinman et al., 2013), multiple sclerosis-related demyelination (Hamada and Kole, 2015; Radecki et al., 2018), and neuropathic pain (Shiers et al., 2018). This suggests altered AIS geometry could be a shared mechanism of CNS dysfunction.

Patients with type 2 diabetes show slight decrement of cognitive performance across their lifespan and have increased risk of developing Alzheimer's-like dementia (Biessels et al., 2014; Biessels and Despa, 2018). Some studies suggest that alteration of the AIS in brain regions important for cognition may change cognitive behavior in type 2 diabetes and related conditions. For example, concurrent cortical AIS shortening and cognitive impairment are present in type 2 diabetic $\mathrm{db} / \mathrm{db}$ mice (Yermakov et al., 2018, 2019) and Alzheimer's-like mice (Marin et al., 2016; Sri et al., 2019). In another study of mice, pharmacological treatment of neuropathic pain led to simultaneous reversal of cognitive impairment and AIS shortening in the infralimbic cortex (Shiers et al., 2018), supporting a link between AIS shortening in cortical neurons and

Received May 6, 2021; accepted September 7, 2021; First published September 16, 2021.

The authors declare no competing financial interests.

Author contributions: R.B.G., D.V.M.N., L.M.Y., C.G.-I., P.W., and K.S. designed research; R.B.G., D.V.M.N., L.M.Y., J.M.J., J.N.S., J.K.S., and J.A.M. performed research; R.B.G., D.V.M.N., L.M.Y., J.M.J., J.N.S., J.K.S., and J.A.M. analyzed data; R.B.G., P.W., and K.S. wrote the paper.

This work was supported by NIH Grants R01NS107398 (to K.S.), R03NS112981 (to K.S.), and F30NS124237 (to J.N.S.).

Acknowledgements: We thank Mike Bottomley (Statistical Consulting Center, Wright State University) for help with experimental design and statistical analysis; Domenica Drouet, Dr. Andrew Koesters, Dr. Mark Rich, Dr. Kathy Engisch, and Dr. Eric Bennett (Wright State University) for help with carrying out experiments, providing essential equipment, and critical feedback; Dr. Thomas Fleming, Dr. Sebastian Brings, and Dr. Peter Nawroth (University of Heidelberg) for providing the Rat anti-MG-H1 1D2 tissue culture supernatant unpurified antibody and critical feedback; and the lab of Dr. Peter Wenner (Emory University) for critical feedback.

Correspondence should be addressed to Ryan B. Griggs at ryan. griggs@wright.edu or Keiichiro Susuki at keiichiro.susuki@wright.edu.

https://doi.org/10.1523/ENEURO.0201-21.2021

Copyright $(2021$ Griggs et al.

This is an open-access article distributed under the terms of the Creative Commons Attribution 4.0 International license, which permits unrestricted use, distribution and reproduction in any medium provided that the original work is properly attributed. cognition. To better understand the mechanisms underlying cognitive impairment in type 2 diabetes and related conditions, it is important to identify the factor(s) that initiate changes in AIS geometry in cortical neurons. In $\mathrm{db} / \mathrm{db}$ mice, exercise treatment concomitantly ameliorated hyperglycemia and AIS shortening associated with type 2 diabetes (Yermakov et al., 2018), suggesting that type 2 diabetes-related factors such as high-glucose or insulin resistance may alter AIS geometry. Another potential mediator of AIS shortening in type 2 diabetes is the reactive carbonyl species and glucose metabolite methylglyoxal. We recently reported that methylglyoxal disrupts AnkryinG at paranodes in CNS myelinated axons (Griggs et al., 2018), suggesting that methylglyoxal may also affect the AnkyrinG-containing AIS in cortical neurons. Importantly, disruption of methylglyoxal metabolism, including pathophysiological increase of methylglyoxal or methylglyoxal-derived advanced glycation endproducts, in humans is linked to the same neurological conditions as altered AIS geometry in animal models, including type 2 diabetes (Andersen et al., 2018), Alzheimer's disease (Kuhla et al., 2007; Haddad et al., 2019), aging (Kuhla et al., 2006; Beeri et al., 2011; Srikanth et al., 2013), multiple sclerosis (Wetzels et al., 2019), and pain (Düll et al., 2019). The effect of methylglyoxal, glucose, or insulin on AIS geometry and the operation of neuronal networks is unknown.

To determine the key type 2 diabetes-related factor that leads to AIS shortening and test the hypothesis that this AIS shortening is associated with reduced neuronal activity at the cellular and network levels we used a reductionist approach. We exposed dense cultures of dissociated postnatal mouse cortex to modified levels of insulin, glucose, or methylglyoxal and quantified AIS geometry using AnkyrinG and $\beta$ IV spectrin immunofluorescence. Since increase of methylglyoxal, but not insulin or glucose, was sufficient to shorten the AIS, we further characterized the effect of methylglyoxal on our cultures by assessing cell viability, the reversibility of AIS shortening, and the celltype specificity of AIS shortening using the excitatory neuron marker $\mathrm{Ca}^{2+} /$ calmodulin-dependent protein kinase $\mathrm{Il} \alpha$ $(\mathrm{CaMKII} \alpha)$. Moreover, we evaluated the temporal relationship between methylglyoxal-evoked changes in AIS length, single-cell excitability, and neuronal network activity. We are the first to uncover that short-term elevation of methylglyoxal, a metabolic factor whose pathophysiological increase is implicated in many neurological disease states including type 2 diabetes, changes AIS geometry and transiently alters neuronal function at both the singlecell and network level.

\section{Materials and Methods}

\section{Animals}

Adult male and female C57BL/6J mice (The Jackson Laboratory; RRID:IMSR_JAX:000664) aged 7-30 weeks were used for in-house breeding for postnatal cortical cultures. Mice were housed in Laboratory Animal Resources at Wright State University at $21-25^{\circ} \mathrm{C}$ and $20-60 \%$ humidity under $12 / 12 \mathrm{~h}$ light/dark conditions (lights on 7 A.M. to 
7 P.M.) with ad libitum access to food and water. Mice were provided wood-chip bedding, toilet paper roll cardboard tubes, shredded accordion paper, and thick cotton pads for enrichment and group housed except when single housing was required for breeding purposes. All animal procedures were approved by the Institutional Animal Care and Use Committee at Wright State University (Animal Use Protocol \#1113 and \#1190) and conducted in accordance with the National Institutes of Health Office of Laboratory Animal Welfare Guide for the Care and Use of Laboratory Animals and the ARRIVE 2.0 guidelines (Percie du Sert et al., 2020).

\section{Postnatal cortical cultures}

Preparation and culturing of dissociated cells from postnatal mouse cortices was similar to as described previously (Beaudoin et al., 2012) using the following materials: Neurobasal-A Medium (10888-022, Thermo Fisher Scientific), Neurobasal Plus Medium (A3582901, Thermo), Neurobasal-A without D-glucose or sodium pyruvate (A2477501, Thermo), B-27 Supplement (17504-044, Thermo), B-27 Plus Supplement (A3582801, Thermo), B-27 without insulin (A1895601, Thermo), GlutaMAX Supplement (35050061, Thermo), sodium pyruvate (11360070, Thermo), HibernateA Medium (A1247501, Thermo), gentamycin (G1272, Sigma-Aldrich), papain (PDS kit: LK003178, Worthington Biochemical Corporation), DNase I (90083, Thermo), fetal bovine serum (FBS; 26140-087, Thermo), poly-L-lysine (P2636, Sigma), coverslips precoated with poly-L-lysine (12 mm \#1.5 Biocoat 354085, Corning; or GG-12-1.5-PLL, Neuvitro Corporation), Biosafety Cabinet Class II A2 (NU540-500, Nuaire), $\mathrm{CO}_{2}$ incubators (NU-5710, Nuaire).

\section{Preparation of dissociated cortical cells}

Plating media (Neurobasal-A or Neurobasal Plus, $2 \mathrm{~mm}$ GlutaMAX, 2\% B-27 or 2\% B-27 Plus, $5 \%$ FBS, $10 \mu \mathrm{g} / \mathrm{ml}$ gentamycin) and growth media (Neurobasal-A or Neurobasal Plus, 2 mm GlutaMAX, 2\% B-27 or 2\% B-27 Plus) were prepared fresh, stored at $4^{\circ} \mathrm{C}$, and used within $7 \mathrm{~d}$. Papain (40 $\mathrm{U} / \mathrm{ml}$ ) was solubilized in Neurobasal-A or Hibernate-A Medium containing DNase I $(40 \mathrm{U} / \mathrm{ml})$ and prewarmed at $37^{\circ} \mathrm{C}$ for $20 \mathrm{~min}$. Whole brains were removed from decapitated male and female mouse pups at postnatal day (P)0 and covered in ice-cold Hibernate-A. The left and right cortices were separated, meninges removed, minced into $\sim 2-\mathrm{mm}^{3}$ pieces and transferred to a sterile 14-ml culture test tube containing 1-ml ice-cold Hibernate-A. Cells were enzymatically and mechanically dissociated by adding $1 \mathrm{ml}$ of prewarmed, activated papain/DNase solution (20 U/ml final concentration), incubating in a $37^{\circ} \mathrm{C}$ bead bath for 20 min with gentle swirling every $5 \mathrm{~min}$, followed by gentle trituration three to five times using a fire-polished 9-inch Pasteur pipet. Triturated solution containing cells and tissue debris was passed through a sterile $100-\mu \mathrm{m}$ strainer, transferred to a $15-\mathrm{ml}$ polystyrene centrifuge tube, centrifuged $5 \mathrm{~min}$ at $250 \times \mathrm{g}$, supernatant decanted, cell pellet resuspended in $\sim 1$ to 2 -ml plating media per cortical pair, cells counted using a hemocytometer (0267110, Thermo), and concentration adjusted to $1 \times 10^{6}$ $\mathrm{cells} / \mathrm{ml}$ with additional plating media. Preparation of dissociated cells from both cortices from one postnatal mouse pup brain typically yielded circa $2 \times 10^{6}$ cells; however, sometimes cells dissociated from multiple pup brains were combined into one tube if larger numbers of cells were needed for a given experiment, and this was deemed as one culture preparation, or simply one culture. For whole-cell patch clamp electrophysiology, cultures were prepared as above but from embryonic day (E)18 cortices (C57ECX, BrainBits LLC) using Neurobasal Medium (21103049, Thermo) and Hibernate-E (A1247601, Thermo).

\section{Plating}

Coverslips (12 mm), tissue culture dishes (35 mm), and multielectrode arrays (MEAs) were precoated with poly-Llysine diluted to $0.1 \mathrm{mg} / \mathrm{ml}$ in $0.1 \mathrm{M}$ borate buffer $\mathrm{pH} 8.5$ and incubated $>1 \mathrm{~h}$ to overnight at $37^{\circ} \mathrm{C}$, washed with sterile water 2 times, allowed to completely dry in biosafety cabinet, and stored for up to two weeks at $4^{\circ} \mathrm{C}$ until use. Dissociated cortical cells from P0 pups were pipetted onto precoated: $12-\mathrm{mm}$ coverslips contained in $35-\mathrm{mm}$ dishes $(100,000$ cells in $100 \mu \mathrm{l}$ per coverslip, three to four coverslips per 35-mm dish) for immunofluorescence and cell viability assays, $35-\mathrm{mm}$ dishes $(1,000,000$ cells in $1 \mathrm{ml})$ for Western blotting, or MEAs $(50,000$ cells in $50 \mu \mathrm{l})$ for neuronal network activity recordings, allowed to settle onto the substrate for at least $10 \mathrm{~min}$, and plating media added up to a final volume of $2 \mathrm{ml}$ (35-mm dishes) or $1 \mathrm{ml}$ (MEAs). After overnight incubation, all the plating media was exchanged for growth media and cultures were maintained in growth media with $50 \%$ media changes every $3-4 \mathrm{~d}$ for coverslips/dishes or up to $7 \mathrm{~d}$ for MEAs where specialized membrane lids were used to prevent evaporation (ALAMEAMEM5, ALA Scientific). For whole-cell patch clamp electrophysiology 50,000 dissociated cortical cells from E18 pups were plated on 22-mm coverslips (C022001, Matsunami Glass USA Inc.) precoated with FBS, poly-L-lysine, and laminin at a similar cell density to the 12-mm coverslips used for immunofluorescence analyses.

\section{Characterization}

Previous studies indicate that the AIS matures during the first week of culture and maximal levels of both AnkyrinG (Galiano et al., 2012) and Nav (Yang et al., 2007) protein are present circa day in vitro (DIV)7. Therefore, drug exposure experiments were initiated on or after DIV8. The percentage of microtubule associated protein 2 (MAP2) positive neurons MAP2 + $(78.2 \pm 2.5 \%)$ and glial fibrillary acidic protein (GFAP) positive astrocytes (21.8 \pm $2.5 \%)$ at DIV8 was similar to the percentage of MAP2+ $(72.6 \pm 3.4 \%)$ and GFAP $+(27.4 \pm 3.4 \%)$ cells at DIV14 ( $n=12$ coverslips from four cultures). This distribution of astrocytes $(\sim 20 \%)$ is greater than the $6-8 \%$ previously reported in low density cortical cultures prepared from postnatal P0 to P1 (Beaudoin et al., 2012) or embryonic E17 (Bélanger et al., 2011) mice. The percentage of MAP2+ neurons that were $\mathrm{CaMKII} \alpha+(78.5 \pm 1.07 \%, n=12$ coverslips from three cultures) was greater than CaMKIl $\alpha-$ $(21.5 \pm 1.07 \%, n=12$ coverslips from three cultures) at DIV14. This distribution of putative inhibitory cells ( $20 \%)$ agrees with previous mouse studies of GABA + cells in cortical cultures (Beaudoin et al., 2012) or GAD67+ cells in hippocampal cultures (Prestigio et al., 2019). The density of cells in 
our cortical cultures (empirically determined to be $\sim 1400$ cells $/ \mathrm{mm}^{2}$ ) is about three times the density of a previous study of AIS plasticity in hippocampal cultures where 45,000 cells were seeded onto 13-mm coverslips (calculated density of $\sim 440$ cells $/ \mathrm{mm}^{2}$; Evans et al., 2013). This is notable, since a previous study in rat embryonic hippocampal cultures indicates that cell density alters neuronal excitability and AIS geometry (Guo et al., 2017).

\section{Drug exposure}

Dissociated PO cortical cells were cultured on coverslips contained in 35-mm dishes for immunofluorescence or directly in 35-mm dishes for Western blotting. Cultures were exposed to insulin (A11382II, Thermo), D-(+)-glucose (G8270, Sigma), D-mannitol (M4125, Sigma), methylglyoxal (6.49 M stock, M0252, Sigma), or tetrodotoxin (TTX; T-550; Alomone Labs) by dilution of the drug in growth media to $2 \times$ final concentration, syringe-filtration through $0.22-\mu \mathrm{m}$ PES membranes (229746, CELLTREAT Scientific Products), prewarming to $37^{\circ}$ $\mathrm{C}$, and then adding $1 \mathrm{ml}$ to $35-\mathrm{mm}$ dishes containing $1 \mathrm{ml}$ of conditioned medium for a $1 \times$ final concentration.

\section{Insulin exposure}

Insulin resistance was generated in our postnatal cortical cultures as previously described in embryonic mouse cortical cultures (Kim et al., 2011) with modification. Cultures were grown in growth media containing standard Neurobasal-A from DIV0 to DIV10. Since the concentration of insulin in standard Neurobasal-A media is already supraphysiological $(\sim 700 \mathrm{~nm})$, we generated insulin resistance by first omitting insulin completely for $24 \mathrm{~h}$ at DIV10-DIV11 followed by $24 \mathrm{~h}$ of exposure at DIV11DIV12 to standard growth media containing $\sim 700 \mathrm{~nm}$ insulin or growth media modified to contain 0,20 , or $100 \mathrm{~nm}$ insulin. We validated the generation of insulin resistance by challenging a subset of cultures grown on $35-\mathrm{mm}$ dishes with $20 \mathrm{~nm}$ insulin for $15 \mathrm{~min}$ followed by quantification of the phosphorylated to non-phosphorylated Akt ratio (pAkt/Akt) using Western immunoblotting band density. Cultures used to quantify AIS length did not undergo the 15-min insulin challenge.

\section{Glucose exposure}

To model hyperglycemia in mature (DIV10-DIV11) cultures, coverslips were grown in standard culture conditions from DIV0 to DIV10 and then exposed to unmodified media containing $25 \mathrm{~mm}$ glucose or media modified to contain $50 \mathrm{~mm}$ high glucose or $25 \mathrm{~mm}$ glucose plus $25 \mathrm{~mm}$ mannitol. Mannitol was added to control for potential effect of hyperosmolality generated by doubling the amount of glucose in standard culture conditions ( $25 \mathrm{~mm}$ ) to $50 \mathrm{~mm}$ high glucose.

\section{Methylglyoxal exposure}

Cultures were grown in standard growth media followed by methylglyoxal exposure initiated on DIV13 for Western immunoblotting and MEA analyses, DIV8-DIV13 for immunofluorescence quantitation of AIS geometry or viability, and DIV12-DIV14 for whole-cell electrophysiology.

\section{Western blotting of postnatal cortical cultures grown on 35-mm dishes}

Dissociated cells from P0 mice cortices were grown on poly-L-lysine coated 35-mm tissue culture treated dishes as described above. Protein lysates for Western immunoblotting were prepared by rinsing dishes in ice-cold PBS twice then adding ice-cold lysis buffer $(25 \mathrm{~mm}$ Tris $\mathrm{HCl}$ at $\mathrm{pH}$ 7.5, $150 \mathrm{~mm} \mathrm{NaCl}, 1 \%$ Triton $\mathrm{X}-100,0.5 \%$ deoxycholate, $0.1 \%$ SDS, and $10 \mathrm{~mm}$ EDTA) containing protease inhibitor cocktail (P8340, Sigma) and phosphatase inhibitor cocktail (78428, Thermo). Cells were collected using a sterile cell scraper (08-100-241, Fisher) in $2 \times 100 \mu \mathrm{l}$ volumes of ice-cold lysis buffer, transferred to ice-cold 1.5$\mathrm{ml}$ tubes, vortexed, incubated on ice for $10 \mathrm{~min}$, then centrifuged at $18,000 \times g$ for $10 \mathrm{~min}$ at $4^{\circ} \mathrm{C}$ in a Sorvall Legend Micro 21R centrifuge (Thermo). After centrifugation, supernatant was collected into fresh, ice-cold tubes and protein concentrations were measured using a Pierce BCA Protein Assay (232525, Thermo) or Coomassie Plus Bradford Assay (23236, Thermo). Samples (10- $\mu \mathrm{g}$ protein) were denatured at $95^{\circ} \mathrm{C}$ for $5 \mathrm{~min}$ in $4 \times$ Laemmli sample buffer (1610747, Bio-Rad) or Novex NuPAGE LDS sample buffer (NP0007, Thermo) with $\beta$-mercaptoethanol (1610710, Bio-Rad) or 10× reducing agent (DTT; B0009, Thermo), then run on either a 4-20\% Mini-PROTEAN TGX stain-free gel (4568096, Bio-Rad) or on 4-12\% gels using the Novex Bolt mini-gel system (NW0412B, B1000B, BT000614, Thermo). The gel was then transferred to nitrocellulose membrane with $0.45 \mu \mathrm{m}$ pore size (1620115, Bio-Rad). Membranes were blocked for $1 \mathrm{~h}$ in Pierce protein-free (PBS) blocking buffer (37572, Thermo) for MG-H1 or 20 mm Tris, pH 8.0 and $0.05 \%$ (v/v) Tween 20 (TBST) containing 4\% (w/v) milk for all other antigens. Membranes were incubated overnight at $4^{\circ} \mathrm{C}$ in primary antibody diluted $(1: 1000)$ in TBST with milk or proteinfree blocking buffer. Primary antibody was washed $3 \times 5$ min in TBST followed by incubation of the membrane in horseradish peroxidase (HRP)-conjugated secondary antibody (1:10,000 or 1:20,000; Jackson ImmunoResearch) for $1 \mathrm{~h}$ at room temperature. Signals generated by Pierce ECL Plus Western Blotting Substrate (32132, Thermo) were detected using a ChemiDoc MP Imaging System (Bio-Rad) or Azure 600 (Azure Biosystems). Quantification of the band density was performed using ImageLab software (Bio-Rad). The densities of the bands of interest were normalized to the relative expression of GAPDH or Total Protein staining by TGX stain-free precast gels (4568096, Bio-Rad), SYPRO Ruby (S4942, Sigma), or Azure Ponceau (10147-344, VWR).

\section{Immunofluorescent analysis of postnatal cortical cultures grown on 12-mm coverslips}

Immunostaining of postnatal cortical cultures was performed as follows. Custom humidity chambers were created by super gluing caps from 1.5-ml microcentrifuge tubes to the bottom of $100-\mathrm{mm}$ Petri dishes and adding a piece of Kimwipe wetted with $\mathrm{dH}_{2} \mathrm{O}$. After drug exposure, media was removed and dishes were rinsed three times in prewarmed $1 \times$ PBS (137 mM NaCl, $2.7 \mathrm{~mm} \mathrm{KCl}$, and 11.9 mm phosphate) then fixed in room temperature $4 \%$ paraformaldehyde (PBS, $\mathrm{pH}$ 7.4) for 20 min. After washing $3 \times 5 \mathrm{~min}$ in PBS, coverslips were taken from the $35-\mathrm{mm}$ 
Table 1: Primary antibodies used for immunofluorescent imaging or Western immunoblotting

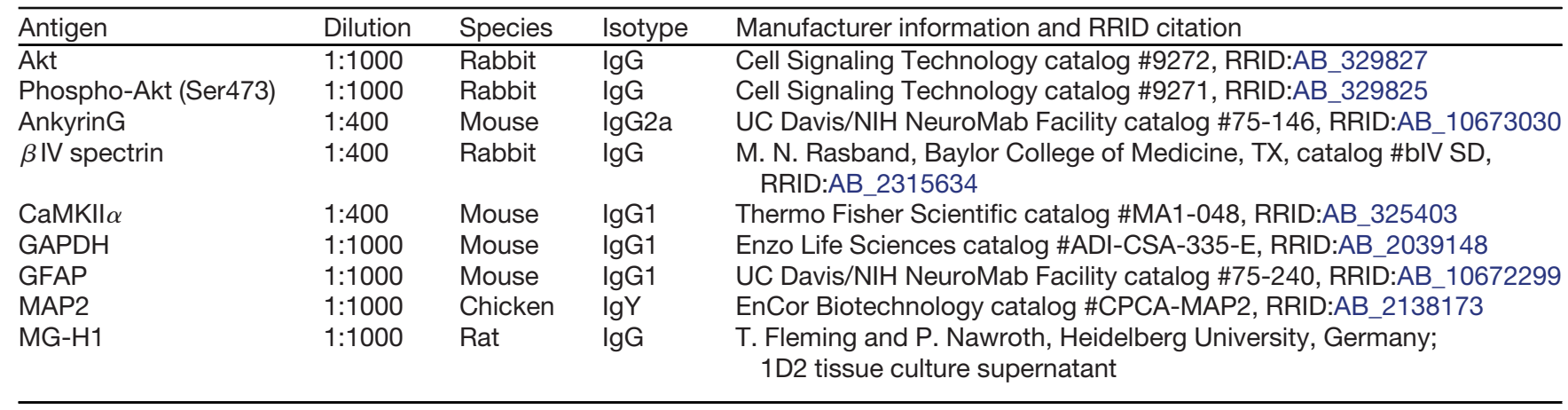

dishes and dabbed on a Kimwipe to remove liquid then placed onto the microcentrifuge caps within the humidity chambers to complete the immunostaining process. Coverslips were blocked in PBSTGS $(1 \times$ PBS, $0.1 \%$ Triton X-100, 10\% goat serum) for 30-60 min. Fifty microliters of primary antibody diluted in PBSTGS was added to each coverslip and then coverslips within the custom humidity chambers were incubated in primary antibody overnight at $4^{\circ} \mathrm{C}$. After $3 \times 5 \mathrm{~min}$ wash in PBSTGS, appropriate secondary antibodies were diluted in PBSTGS, added to the coverslips, and incubated in the dark at room temperature for $1 \mathrm{~h}$. Coverslips were washed for $5 \mathrm{~min}$ each in PBSTGS, $0.01 \mathrm{~m}$ phosphate buffer, $0.005 \mathrm{~m}$ phosphate buffer, allowed to air dry, then mounted on non-coated slides using either KPL Mounting Medium (71-00-16, VWR) or ProLong Gold Antifade Mountant without DAPI (36934, Thermo) or with DAPI (P36941, Thermo). In some instances, Hoechst was added during the PBSTGS wash step after secondary antibody incubation. After mounting onto slides, coverslips were secured using clear nail polish and stored at $-20^{\circ} \mathrm{C}$ until imaging analysis.

The primary antibodies used are listed in Table 1. AffiniPure minimally cross-reactive secondary antibodies used for immunofluorescent imaging were conjugated to Alexa Fluor $(594,488,350)$ or AMCA (Jackson ImmunoResearch). Secondary antibodies used for western immunoblotting were conjugated to HRP (Jackson ImmunoResearch). Hoechst or DAPI were used to stain cell nuclei.

Images of immunostained coverslips were obtained using a fluorescence microscope (Axio Observer Z1 with Apotome 2 fitted with AxioCam Mrm CCD camera, Carl Zeiss Microscopy LLC) and measurement of AIS length was performed using the curve spline measurement tool in ZEN2.0 (Carl Zeiss) or a MATLAB script adapted from previous studies (Grubb and Burrone, 2010; Yau et al., 2014). Our comparison of AIS length measured by eye versus by custom MATLAB script yielded similar results, which is consistent with a strong correlation (Spearman $r>0.8$ ) of AIS length measured by eye with AIS measurement using custom-written MATLAB scripts as reported previously (Grubb and Burrone, 2010; Evans et al., 2015; Guo et al., 2017; Fruscione et al., 2018; Zhang et al., 2019). Measurement of AIS start location (distance from the edge of the soma to the start of the AIS) was performed as described previously (Guo et al., 2017). Only continuous, non-overlapping AISs originating from a MAP2 + cell were quantified by an observer blinded to experimental treatment.

\section{Cell viability assay}

Cell viability was determined using a commercially available dual-wavelength fluorescent kit (LIVE/DEAD viability/cytotoxicity assay kit: L3224, Invitrogen). Live cells are detected by intracellular esterase activity using the dye calcein-AM ( $\sim 495 / 515 \mathrm{~nm}$ ex/em; green). Dead cells are detected by a large increase in fluorescence of the dye ethidium homodimer-1 when bound to nucleic acids, which only occurs if the plasma membrane is compromised ( $495 / 635 \mathrm{~nm}$ ex/em; red). Optimal dilution (1:2000 for each dye) of calcein-AM (4 mM in DMSO) and EthD-1 (2 mM in DMSO: $\mathrm{H}_{2} \mathrm{O}$ 1:4) stocks was empirically determined by comparing signal:noise ratios in the green and red channels. We validated the live/dead assay by exposing mature cortical cultures to either $1 \times$ PBS or $100 \%$ methanol for $15 \mathrm{~min}$ and confirming methanol-induced cell death. After drug exposure, coverslips were rinsed $3 \times$ in $1 \times$ PBS and incubated in $80 \mu \mathrm{l}$ of calcein-AM/EthD-1/ PBS solution in the dark at room temperature for $15 \mathrm{~min}$. Excess solution was removed and coverslips were mounted in $5 \mu \mathrm{l}$ calcein-AM/EthD-1/PBS solution, sealed using clear nail polish to minimize evaporation, and immediately imaged to avoid signal decay of calcein-AM fluorescence. The number of cells displaying green or red fluorescence was quantified and data are presented as percentage of number of live cells counted divided by total cells counted (number of live cells + number of dead cells) normalized to media control.

\section{Whole-cell patch clamp recordings}

Patch clamp tight seals (1-3 G $\Omega$ ) were obtained under voltage clamp using thin-walled borosilicate glass capillary (World Precision Instruments) electrodes pulled in two stages using a P-87 Flaming/Brown Micropipette Puller (Sutter Instruments) to obtain microelectrodes with resistances between 6 and $7 \mathrm{M} \Omega$. Once whole-cell configuration was achieved, voltage clamp was maintained at $70 \mathrm{mV}$ for a period of $5 \mathrm{~min}$ to allow stabilization before switching to current clamp configuration to record resting membrane potential. Voltage signals were acquired with an Axopatch 200B patch clamp amplifier (Molecular Devices), 
digitized (Digidata 1200, Molecular Devices), and recorded using Axon pCLAMP 10 (Molecular Devices). Recordings were terminated if a $20 \%$ increase in input resistance was observed. Current clamp recordings were filtered online at $10 \mathrm{kHz}$ and digitized at $20 \mathrm{kHz}$. A liquid junction potential of $-9 \mathrm{mV}$ was experimentally measured and corrected for (Neher, 1992). The patch pipette solution contained the following: $5 \mathrm{~mm} \mathrm{NaCl}, 100 \mathrm{~mm}$ K-gluconate, $36 \mathrm{~mm} \mathrm{KCl,} 10 \mathrm{~mm}$ HEPES, $1.1 \mathrm{~mm}$ EGTA, $1 \mathrm{~mm} \mathrm{MgCl} 2,0.1 \mathrm{~mm} \mathrm{CaCl}$, $1 \mathrm{~mm}$ $\mathrm{Na}_{2}$ ATP, and $0.1 \mathrm{~mm}$ MgGTP with osmolarity of 280-300 mOsm and $\mathrm{pH}$ adjusted to 7.3 with $\mathrm{KOH}$. Recordings were obtained in artificial CSF (aCSF) containing the following: $126 \mathrm{~mm} \mathrm{NaCl}, 3 \mathrm{~mm} \mathrm{KCl}, 2 \mathrm{~mm} \mathrm{CaCl}_{2}, 1.5 \mathrm{~mm} \mathrm{MgSO}_{4}, 1 \mathrm{~mm}$ $\mathrm{NaH}_{2} \mathrm{PO}_{4}, 25 \mathrm{~mm} \mathrm{HEPES}$, and $25 \mathrm{~mm} \mathrm{D}$-glucose with $\mathrm{pH}$ adjusted to 7.4 using $\mathrm{NaOH}$. Drug exposure was performed by infusing control aCSF or aCSF containing drug solution at a similar rate of $3 \mathrm{ml} / \mathrm{min}$. Action potential thresholds were assessed using a current injection step protocol (500-ms duration per step; 20 steps; 10-pA increments; 0-200 pA). A 200 -ms hyperpolarizing current of $20 \mathrm{pA}$ was delivered $800 \mathrm{~ms}$ before every step pulse to determine input resistance and serve as an indicator of the reliability of the step. The current injection step protocol was repeated three times for each cell, and the average value was used for analysis. Rheobase current, threshold voltage and membrane resistance measurements were conducted offline using Clampfit software (Molecular Device). Analysis was performed blinded to experimental treatment.

\section{MEA recording of neuronal network activity}

MEA recordings were performed as previously described (Wagenaar et al., 2006; Hales et al., 2010; Fong et al., 2015) using the MEA2100-60-System (Multi Channel Systems) that includes MEA2100-HS60 headstage with integrated heating element, MCS-interface board 3.0 multiboot, Multi Channel Suite acquisition and analysis software, and temperature controller. MEAs (60MEA200/ 30iR-Ti-g, Multi-Channel Systems) were precoated with poly-L-lysine as described above, a $25-\mu$ l drop of $40 \mu \mathrm{g} /$ ml laminin (L2020, Sigma) was carefully pipetted directly onto the center of the electrode array, and MEAs were incubated at $37^{\circ} \mathrm{C}$ during preparation of postnatal cortical cells. Before plating the cells, laminin was incompletely aspirated leaving a wetted area that localizes dispersion of the cell suspension on top of the electrode array. MEAs were maintained by $50 \%$ media changes every 3-7 d with fresh prewarmed growth media using specialized gaspermeable membrane ALA lids (ALAMEA-MEM5, ALA Scientific Instruments) to prevent evaporation that can result in increased tonicity and toxicity (Potter and DeMarse, 2001). MEAs remained in the incubator during the experiment except during the 15 min recording period at each time point. Recordings were performed with the MEA2100-60-System on the benchtop in ambient air with headstage heated to $37^{\circ} \mathrm{C}$. The ALA lids prevented contamination during repeated recordings across several days. Since we observed that $50 \%$ media changes altered spiking activity within the first several hours, experimental recordings were performed the day after media changes. Vehicle control $(2-\mu l$ growth media) or methylglyoxal $(2 \mu \mathrm{l}$ of $500 \times$ final concentration) were added to MEAs containing 1 -ml conditioned media resulting in a $1 \times$ final concentration. The $\mathrm{pH}$ was similar before and after addition of either media or methylglyoxal. Recordings were digitized at $20,000 \mathrm{~Hz}$. Threshold for spike detection was set at mean noise \pm 5 SDs on a per channel basis as previously reported (Charkhkar et al., 2015). Multi Channel Suite (Multi Channel Systems) and Neuroexplorer 5.0 (Nex Technologies) were used to create raster plots and extract network spiking and bursting parameters.

MEA-wide network spike frequency was obtained by dividing the recording duration $(15 \mathrm{~min})$ into the total amount of spikes from 59 channels (reference channel was omitted; Wagenaar et al., 2006; Fong et al., 2015). Network bursts were extracted using the MCS Analyzer Burst Analyzer tool with settings of 50-ms maximum interval to start/end burst, 100-ms minimum interval between bursts, $50-\mathrm{ms}$ minimum duration of burst, at least five spikes within a burst, and at least 15 channels participating in the network burst. MEA-wide network bursting, defined as synchronized spiking activity across all or most of the recording channels, was consistently seen in all MEA preparations. The variance of network spike frequency in the MEAs at baseline was relatively large; therefore, results are presented as percentage of baseline network spike frequency within each MEA. To account for heterogeneity of network function between cultures as previously reported (Wagenaar et al., 2006), we used replicate (sister cultures) MEAs from multiple cultures.

\section{Experimental design and statistical analysis}

Statistics for both $n=A I S / i m a g e / c o v e r s l i p$ and $n=$ culture preparation (average of AIS geometry, cell viability, or cell distribution outcomes within a single culture preparation) are reported in the results text. Comparison of the means between two groups was performed using unpaired, homoscedastic $t$ test. Effect of insulin, glucose, or methylglyoxal concentration was analyzed using one-way ANOVA and/or linear regression. An interaction of time and drug was determined using two-way ANOVA. Multiple comparison post hoc tests are listed in the results text. An $\alpha$ value of $\alpha=0.05$ was used to determine statistical significance. All data were analyzed using Prism 9.1 (GraphPad) or SAS 9.4 (SAS Institute). Data for $\mathrm{n}=\mathrm{AlS}$ are presented as cumulative frequency distributions or scatter plots where the width of the distribution of data points is proportional to the number of data points at a given $Y$ value. Data for $n=$ culture preparation (larger open symbols) are overlayed on the scatter plots showing each AIS, image, or coverslip (small gray dots). Figures show mean \pm SEM and the $p$ value or "ns" (not significant) for $n=$ culture preparation (immunofluorescence) or $\mathrm{n}=\mathrm{MEA} /$ cell (electrophysiology).

\section{Results}

\section{Insulin sensitivity manipulation does not affect AIS length}

Type 2 diabetes is canonically characterized by insulin resistance and elevated glucose. First, we tested whether altering insulin sensitivity in postnatal cortical cultures 


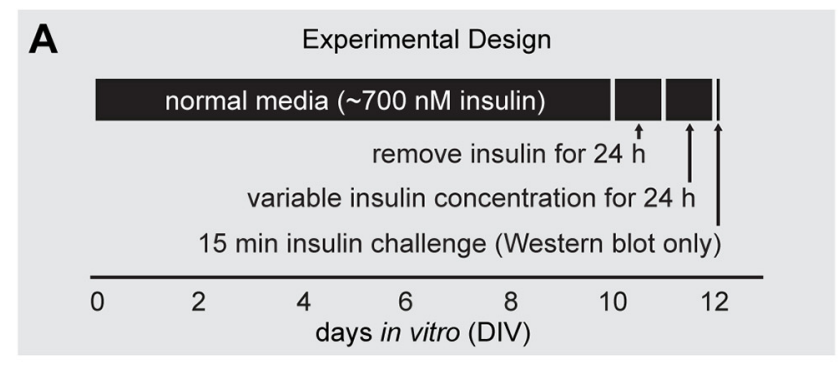

B
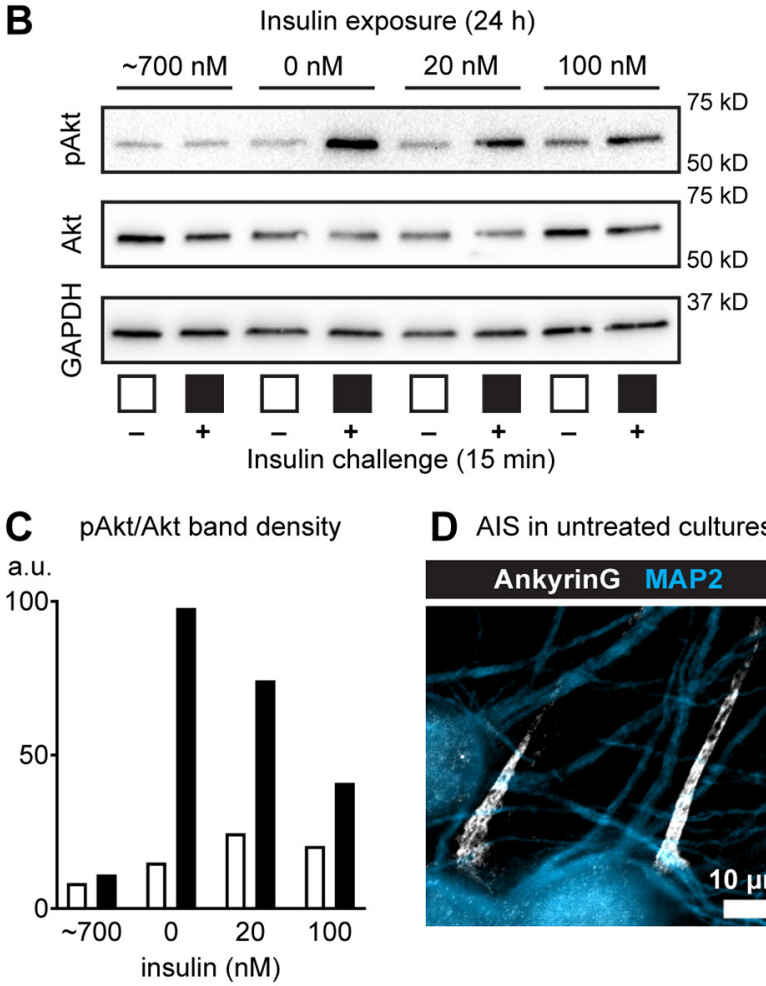

D AIS in untreated cultures

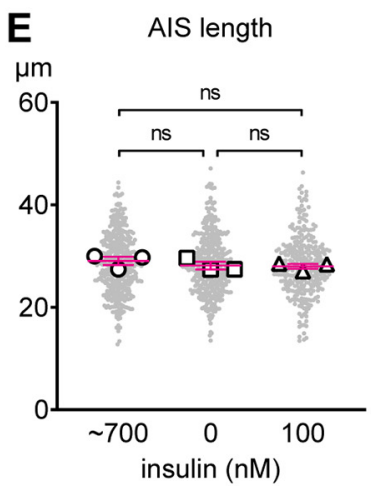

F Cumulative fraction

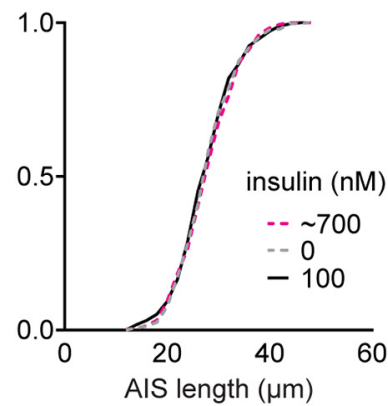

Figure 1. Effect of altering insulin on AIS length in mouse cortical cultures. $\boldsymbol{A}$, Schematic showing the experimental design and timeline of variable insulin exposure to induce insulin resistance (revealed by Western blotting after a 15-min challenge to insulin) in culture. $\boldsymbol{B}, \boldsymbol{C}$, Western immunoblotting results showing phosphorylation status of Akt after inducing acute insulin resistance. White bars denote absence of 15-min insulin challenge and black bars denote presence of 15-min insulin challenge that was used to reveal insulin sensitivity status. a.u. (arbitary units). $\boldsymbol{D}$, Representative image of mature postnatal cortical cultures at DIV10 showing MAP2 (somatodendritic affects AIS length. We used a protocol previously established to produce insulin resistance in embryonic mouse primary cortical cultures (Kim et al., 2011). As shown in the experimental design (Fig. 1A), we removed insulin for $24 \mathrm{~h}$ in mature cultures, varied the insulin concentration for an additional $24 \mathrm{~h}$, then validated the presence of insulin resistance via Akt immunoblotting and quantified AIS length by immunostaining for AnkryinG, the master organizer of the AIS (Rasband, 2010). Previous studies suggest that a $24 \mathrm{~h}$ period of time might be sufficient to observe changes in AIS geometry in cultured CNS neurons (Grubb and Burrone, 2010; Chand et al., 2015). Quantification of phosphorylated Akt and total Akt immunoblot band density revealed that increasing the concentration of insulin for $24 \mathrm{~h}$ led to a lower pAkt/Akt ratio when challenged with insulin for $15 \mathrm{~min}$ (Fig. 1B,C). Decreased phosphorylation of Akt is indicative of insulin resistance. A representative image of the AIS (AnkyrinG) and the somatodendritic domain (MAP2) in untreated mature cultures is shown (Fig. 1D). AlS length was similar after $24 \mathrm{~h}$ of exposure to $700 \mathrm{~nm}(28.61 \pm 0.28 \mu \mathrm{m}, n=416$ AIS from three cultures), $0 \mathrm{~nm}(28.41 \pm 0.29 \mu \mathrm{m}, n=396$ AIS from three cultures), or $100 \mathrm{~nm}(28.20 \pm 0.34 \mu \mathrm{m}, n=293$ AIS from three cultures) insulin (insulin concentration: $F_{(2,1102)}=0.45, p=0.64$; Tukey) when $\mathrm{n}=$ AIS. When analyzed at the culture preparation level, AIS length was also similar (insulin concentration: $F_{(2,6)}=0.68, p=0.54$; Tukey; $n=3$ cultures; Fig. 1E). Cumulative frequency plot shows overlapping distributions of AIS lengths after variable insulin exposure (Fig. 1F), indicating that no changes in AIS length are observed in the entire population of cortical neurons.

\section{Glucose does not appreciably change AIS length}

Next, we tested whether elevating glucose levels in mature postnatal cortical cultures changes AIS length. Example images are shown $24 \mathrm{~h}$ after exposing mature cortical cultures to normal Neurobasal-A growth media containing $25 \mathrm{~mm}$ glucose, high-glucose media containing $50 \mathrm{~mm}$ glucose, or normal media containing $25 \mathrm{~mm}$ glucose with $25 \mathrm{~mm}$ mannitol added to control for the elevated osmolarity in high-glucose media (Fig. 2A). When analyzed at the AIS level, AIS length was significantly altered (main effect of treatment: $F_{(2,844)}=3.56, p=0.03$; Tukey) after $24 \mathrm{~h}$ of exposure to $25 \mathrm{~mm}$ glucose $(30.92 \pm 0.37 \mu \mathrm{m}, n=291$ AIS from three cultures), $50 \mathrm{~mm}$ high glucose (29.66 \pm $0.37 \mu \mathrm{m}, n=236$ AIS from three cultures), or $25 \mathrm{~mm}$ glucose plus $25 \mathrm{~mm}$ mannitol $(30.81 \pm 0.32 \mu \mathrm{m}, n=320$ AlS from three cultures). However, when analyzed at the culture preparation

\section{continued}

domain) and Ankyring (AIS). Scale bar: $10 \mu \mathrm{m}$. E, AIS length after inducing conditions of high insulin resistance (normal media containing $\sim 700 \mathrm{~nm}$ insulin), no insulin resistance (0 nM insulin), and moderate insulin resistance (100 nm insulin). Small gray dots indicate each AIS and larger open symbols represent the average AIS length for each culture preparation (293-416 AIS were analyzed from $n=3$ cultures). ns (not significant) indicates statistical comparison at the culture preparation level. $\boldsymbol{F}$, Cumulative fractional distribution of AIS lengths after $24 \mathrm{~h}$ of exposure to $\sim 700,0$, or $100 \mathrm{~nm}$ insulin. 

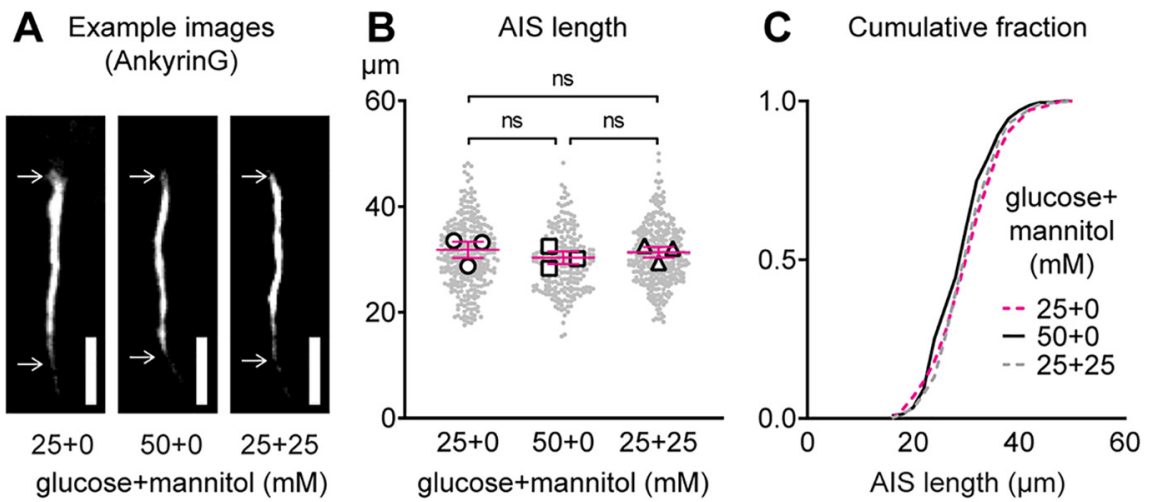

Figure 2. Effect of altering glucose on AIS length in mouse cortical cultures. $\boldsymbol{A}$, Example images of the AIS after $24 \mathrm{~h}$ of exposure to normal culture media containing $25 \mathrm{~mm}$ glucose, high-glucose media containing $50 \mathrm{~mm}$ glucose, or normal media containing 25 mm glucose with $25 \mathrm{~mm}$ mannitol added as an osmotic control in mature cultures (DIV10-DIV11). Scale bar: $10 \mu \mathrm{m}$. B, Quantification of AIS length after $24 \mathrm{~h}$ of exposure to 25,50 , or $25 \mathrm{~mm}$ glucose plus $25 \mathrm{~mm}$ mannitol in mature cultures (DIV10-DIV11). Small gray dots indicate each AIS and larger open symbols represent the average AIS length for each culture preparation (236-320 AIS were analyzed from $n=3$ cultures). ns (not significant) indicates statistical comparison at the culture preparation level. $\boldsymbol{C}$, Cumulative fractional distribution of AIS lengths after $24 \mathrm{~h}$ of exposure to 25,50 , or $25 \mathrm{~mm}$ glucose plus $25 \mathrm{~mm}$ mannitol.

level there was no difference in AIS length (main effect of treatment: $F_{(2,6)}=0.37, p=0.70$; Tukey; $n=3$ cultures; Fig. $2 B$ ). Cumulative frequency distributions show similar AIS length after altering the concentration of glucose or mannitol (Fig. 2C). Taken together, these results show that altered insulin sensitivity (Fig. 1) and high glucose (Fig. 2) do not appreciably change AIS length after $24 \mathrm{~h}$ of exposure in our cortical cultures.

\section{Methylglyoxal exposure increases cellular methylglyoxal-derived hydroimidazolone (MG-H1)}

To test the hypothesis that accumulation of methylglyoxal disrupts AIS geometry, we first needed to determine the concentration of methylglyoxal required to disrupt celIular methylglyoxal metabolism in our in vitro cortical culture model. Reliable estimates of methylglyoxal concentration in the in vivo nervous system during normal, physiological conditions are $1.2 \mu \mathrm{M}$ (Eberhardt et al., 2012) or $40 \mathrm{nmol} / \mathrm{g}$ (Liu et al., 2017) in control dorsal root ganglia, 0.3-1.5 $\mu \mathrm{m}$ (Kurz et al., 2011) or $5 \mu \mathrm{m}$ (Distler et al., 2012) in young adult mouse brain, and $10 \mu \mathrm{m}$ in the cerebrospinal fluid of healthy control middle-aged humans (Kuhla et al., 2005). Conversion of $40 \mathrm{nmol} / \mathrm{g}$ in Liu et al. (2017) to a micromolar concentration assuming a density of DRG tissue of $\sim 1 \mathrm{~g} / \mathrm{ml}$ yields $40 \mu \mathrm{m}$ methylglyoxal. Thus, these studies suggest the normal concentration of methylglyoxal in the brain could be 1-40 $\mu \mathrm{m}$. Other studies report methylglyoxal levels in whole brain as high as 170$360 \mu \mathrm{m}$ (Hambsch et al., 2010), but this may be an overestimation because of inappropriate sample processing (Rabbani and Thornalley, 2014). Information about methylglyoxal levels in CNS tissue during pathophysiological disease conditions such as type 2 diabetes are not wellestablished. Increased methylglyoxal concentration in the blood or serum of patients with type 2 diabetes (Andersen et al., 2018), Alzheimer's disease (Haddad et al., 2019), and aging-related cognitive decline (Beeri et al., 2011) suggest that levels of methylglyoxal may increase in CNS tissues during neurological disease. To validate the concentration of methylglyoxal required to produce pathophysiological disruption of cellular methylglyoxal metabolism in our postnatal cortical cultures, we tested if $24 \mathrm{~h}$ exposure to $0,1,10$, or $100 \mu \mathrm{m}$ methylglyoxal would increase hydroimidazolone (MG-H1), a methylglyoxal-derived advanced glycation end-product (MG-AGE). MG-H1 is a suitable marker for disrupted cellular methylglyoxal metabolism since pathophysiological increase of methylglyoxal leads to accumulation of MG-AGEs in vivo, and upwards of $90 \%$ of MG-AGEs in vivo are the MG-H1 moiety (Rabbani and Thornalley, 2012). MG-H1 and Total Protein in immunoblots of postnatal cortical culture cell lysates are shown (Fig. $3 A$ ). MG-H1 relative band density (normalized to total protein lane volume) was increased by $100 \mu \mathrm{M}$ methylglyoxal $(p=0.001)$ but not 1 or $10 \mu \mathrm{M}$ $\left(F_{(3,8)}=16.08 ; p=0.0009\right.$; Dunnett; $n=3$ cultures; Fig. $\left.3 B\right)$. These results indicate that adding $100 \mu \mathrm{m}$ methylglyoxal for $24 \mathrm{~h}$ is sufficient to acutely disrupt cellular methylglyoxal metabolism in cortical cultures, suggesting that this is a pathophysiologically-relevant dose in our in vitro model.

\section{Characterization of AIS geometry after methylglyoxal exposure}

To test whether methylglyoxal shortens the AIS, we exposed mature neuronal networks to a range of concentrations $(0-100 \mu \mathrm{M})$ in vitro that likely represent physiological to potentially pathophysiological levels for $24 \mathrm{~h}$. Representative images of AnkyrinG and microtubule associated protein 2 (MAP2; somatodendritic marker) show a shorter AIS length after $100 \mu \mathrm{m}$ methylglyoxal exposure for $24 \mathrm{~h}$ (Fig. 4A). AIS length after $24 \mathrm{~h}$ of exposure to $0 \mu \mathrm{M}$ (media control; $28.79 \pm 0.28 \mu \mathrm{m}, n=598$ AIS from 6 cultures), $1 \mu \mathrm{M}(28.39 \pm$ $0.36 \mu \mathrm{m}, n=326$ AIS from three cultures), $10 \mu \mathrm{M}(27.26 \pm$ $0.29 \mu \mathrm{m}, n=489$ AIS from three cultures), or $100 \mu \mathrm{M}$ (25.48 $\pm 0.26 \mu \mathrm{m}, n=522$ AIS from four cultures) methylglyoxal indicate a dose-dependent inverse relationship between concentration of methylglyoxal and AIS length (slope = 

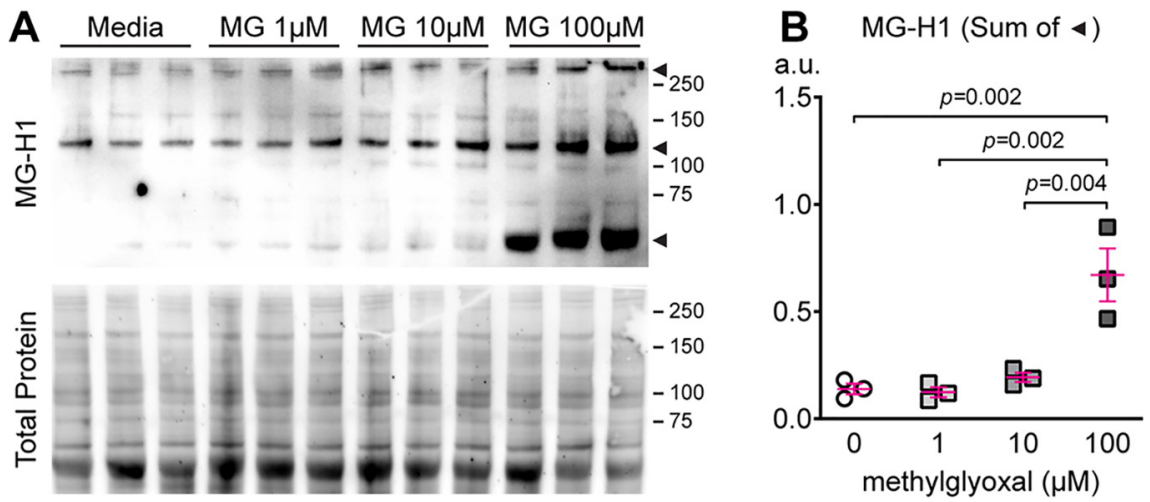

Figure 3. Methylglyoxal increases cellular methylglyoxal-derived hydroimidazolone (MG-H1) in mouse cortical cultures. $\boldsymbol{A}$, Western immunoblot image of cultures exposed to methylglyoxal (0-100 $\mu \mathrm{M} ; 24 \mathrm{~h} ; n=3$ cultures) showing MG-H1 (unpurified rat anti-MG-H1 clone 1D2 antibody) and Total Protein (SYPRO Ruby). B, Quantification of MG-H1 immunoblot density normalized to SYPRO Ruby total protein stain. The sum of band density for the three bands indicated by arrowheads was used for analysis.

$-1.47, R^{2}=0.85, p<0.0001$; linear regression analysis of means) with significant AIS shortening by $10 \mu \mathrm{M}$ $(p=0.0004,5.3 \%$ reduction $)$ and $100 \mu \mathrm{m}(p<0.0001$, $11.5 \%$ reduction) methylglyoxal compared with media only (dose: $F_{(4,2159)}=63.60, p<0.0001$; Dunnett). There was also significant AIS shortening by $100 \mu \mathrm{m}$ $(p=0.001)$ but not $10 \mu \mathrm{M}(p=0.08)$ or $1 \mu \mathrm{M}(p=0.89)$ methylglyoxal when analyzed by culture preparation (dose: $F_{(3,12)}=9.66, p=0.002$; Tukey; $n=3-6$ cultures; Fig. 4B). This $11.5 \%$ AIS shortening induced by $100 \mu \mathrm{m}$ methylglyoxal is about half of the $25 \%$ shortening induced by patterned optogenetic stimulation or high $\mathrm{KCl}$ (Evans et al., 2015) and similar to 8-16\% decrease in AIS length in cortical neurons of $\mathrm{db} / \mathrm{db}$ mice (Yermakov et al., 2018). AIS shortening by $100 \mu \mathrm{m}$ methylglyoxal was generalized across the population of neurons, as indicated by a leftward shift in the cumulative frequency distribution (Fig. 4C).

In addition to AIS length changes, AIS relocation (change in the position of the AIS relative to the soma) is implicated in experimental demyelination (Hamada and Kole, 2015), epilepsy (Harty et al., 2013), and as a mechanism of activity-dependent plasticity that alters intrinsic neuronal function (Grubb and Burrone, 2010; Evans et al., $2013,2017)$. Therefore, we tested whether exposure to $100 \mu \mathrm{m}$ methylglyoxal for $24 \mathrm{~h}$ relocated the start location of the AIS. AIS start location after $24 \mathrm{~h}$ of exposure to media (5.23 $\pm 0.24 \mu \mathrm{m}, n=194$ AIS from three cultures) or $100 \mu \mathrm{m}$ methylglyoxal $(4.70 \pm 0.17, n=246$ AIS from three cultures) was similar when analyzed by AIS ( $p=0.07$, unpaired homoscedastic two-tailed $t$ test) or culture preparation $(p=0.19$, unpaired homoscedastic two-tailed $t$ test; $n=3$ cultures) as shown by scatter plot (Fig. 4D) or cumulative frequency distribution (Fig. 4E). This is consistent with no change in the AIS start location in $\mathrm{db} / \mathrm{db}$ prefrontal cortex (Yermakov et al., 2018).

\section{Methylglyoxal-evoked AIS shortening is not associated with cell death and is reversible}

To rule out the possibility that AIS shortening by methylglyoxal is because of cellular injury we assessed cell viability after methylglyoxal exposure. Live/dead analysis of exposure to methanol, a positive control, or $24 \mathrm{~h}$ of exposure to a supraphysiological dose of $10 \mathrm{~mm}$ methylglyoxal showed substantial cell death (Fig. 5A). However, compared with media, $24 \mathrm{~h}$ of exposure to $100 \mu \mathrm{m}$ methylglyoxal did not affect cell viability when analyzed at the image ( $p=0.33$, unpaired homoscedastic $t$ test; $n=40 \mathrm{im}$ ages from three cultures) or culture ( $p=0.66$, unpaired homoscedastic $t$ test; $n=3$ cultures) level (Fig. 5B). Our results are in agreement with the absence of cell death in cultured embryonic (E17) mouse cortical neurons at DIV14 (Bélanger et al., 2011), DRG neurons (Radu et al., 2012), or SH-SY5Y cells (Nishimoto et al., 2017) after in vitro exposure to $100 \mu \mathrm{m}$ methylglyoxal for $24 \mathrm{~h}$. Similarly in vivo, intraperitoneal injection of methylglyoxal increased the concentration of methylglyoxal in the brain without cytotoxicity in the hippocampus (Distler et al., 2012). In type 2 diabetic $\mathrm{db} / \mathrm{db}$ mice at 10-11 weeks of age, there is elevated free methylglyoxal (Bierhaus et al., 2012) and MG-H1 (Griggs et al., 2019) in serum and AIS shortening without apoptotic cell death in the prefrontal cortex (Yermakov et al., 2018).

A previous study of AIS plasticity in embryonic rat hippocampal neurons indicates that AIS shortening initiated by high $\mathrm{KCl}$ is recoverable when cultures are returned to conditioned media for 24 h (Evans et al., 2015). Thus, we tested whether media replacement would lead to recovery of AIS shortening after an initial $24 \mathrm{~h}$ of exposure to $100 \mu \mathrm{m}$ methylglyoxal. There was a significant interaction of AIS length between media or methylglyoxal exposure and before or after media replacement (drug by time interaction: $F_{(1,1496)}=10.48, p=0.001$; Tukey). Before media replacement, methylglyoxal AIS length $(25.46 \pm 0.35 \mu \mathrm{m}$, $n=383$ AIS from three cultures) was shorter compared with media $(27.9 \pm 0.46 \mu \mathrm{m}, n=274$ AIS from 3 cultures; $p=0.0002$ ). Twenty-four hours after media replacement, AIS length was similar in cultures initially exposed to media (media + media, $27.92 \pm 0.33 \mu \mathrm{m}, n=499$ from three cultures) or methylglyoxal (methylglyoxal + media, $28.10 \pm$ $0.40 \mu \mathrm{m}, n=343$ from 3 cultures; $p>0.99$ ). Similarly at the culture preparation level, the AIS was shorter after the initial $24 \mathrm{~h}$ methylglyoxal exposure before $(p=0.02$; unpaired 
A
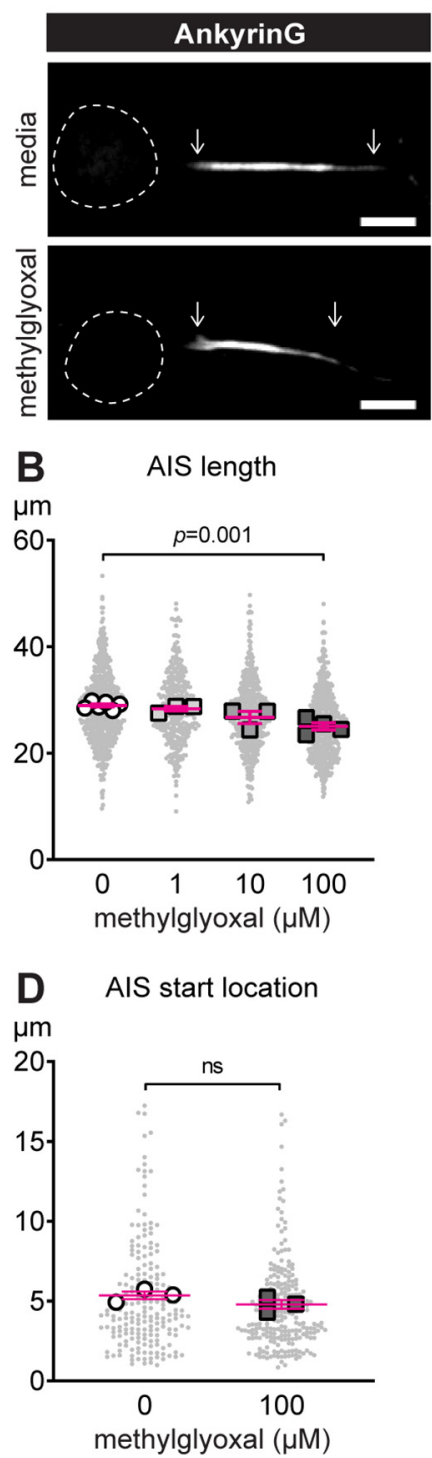

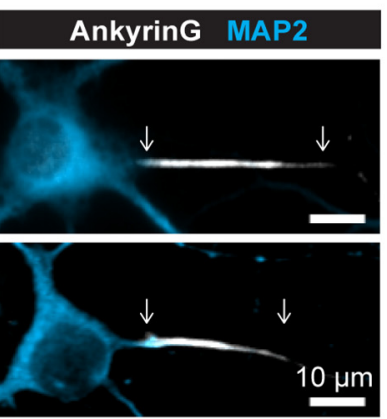

C Cumulative fraction

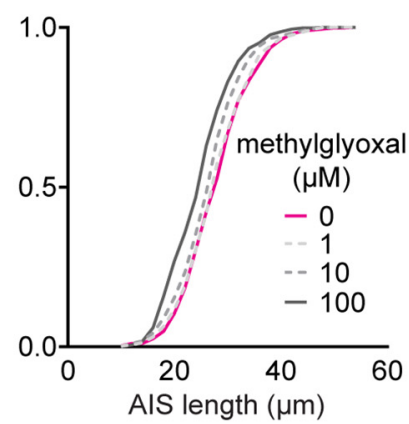

E Cumulative fraction

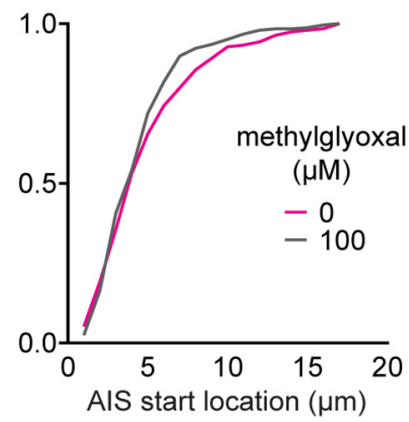

Figure 4. Methylglyoxal reduces AIS length in mouse cortical cultures. A, Ankyring (AIS protein) and MAP2 (somatodendritic protein) immunofluorescence after $24 \mathrm{~h}$ of exposure to media or $100 \mu \mathrm{M}$ methylglyoxal at DIV10-DIV11. Dashed lines indicate the neuronal soma. Scale bar: $10 \mu \mathrm{m}$. B, Quantification of AIS length (Ankyring immunostaining) after $24 \mathrm{~h}$ of exposure to various concentrations of methylglyoxal $(0-100 \mu \mathrm{m})$. Small gray dots indicate each AIS and larger open symbols represent the average AIS length for each culture preparation (326-598 AIS were analyzed from $n=3-6$ cultures); $p$ value indicates statistical comparison at the culture preparation level. $\boldsymbol{C}$, Cumulative fractional distribution of AIS length after $24 \mathrm{~h}$ of exposure to 0$100 \mu \mathrm{m}$ methylglyoxal. $\boldsymbol{D}$, AIS start location (AnkyrinG immunostaining) after $24 \mathrm{~h}$ of exposure to $100 \mu \mathrm{m}$ methylglyoxal. Small gray dots indicate each AIS and larger open symbols represent the average AIS start location for each culture preparation (194246 AIS were analyzed from $n=3$ cultures). ns (not significant) indicates statistical comparison at the culture preparation level. $\boldsymbol{E}$, Cumulative fractional distribution of AIS start location after $24 \mathrm{~h}$ of exposure to $100 \mu \mathrm{m}$ methylglyoxal.

homoscedastic $t$ test; $n=3$ cultures) but not after $(p=0.99$; unpaired homoscedastic $t$ test; $n=3$ cultures) media replacement (Fig. 5C). Cumulative frequency distribution illustrates methylglyoxal-evoked AIS shortening before but not after media replacement (Fig. 5D).

\section{Methylglyoxal shortens the AIS in multiple cell types}

Our results suggest that methylglyoxal produces AIS shortening in the entire population of cortical neurons (Figs. 4C, 5D), whereas previous studies indicate cell type-dependent AIS plasticity in hippocampal or olfactory bulb cultures (Grubb and Burrone, 2010; Evans et al., 2013, 2015; Chand et al., 2015). To test whether methylglyoxal produces AIS shortening in specific types of cortical neurons, we immunostained for MAP2 to label all neurons, $\beta$ IV spectrin to label the AIS, and CaMKII $\alpha$ to identify excitatory (CaMKIl $\alpha+$ ) or putative inhibitory (CaMKII $\alpha-$ ) neurons. Representative images of postnatal cortical cultures at DIV14 double-labeled for MAP2 and CaMKIl $\alpha$ are shown (Fig. 6A). Exposure to $100 \mu \mathrm{M}$ methylglyoxal for $24 \mathrm{~h}$ did not change the distribution of CaMKII $\alpha+/-$ neurons: the mean percentage of all AIS measured that originated from CaMKII $\alpha+$ neurons was similar between media (79.7 $\pm 1.8 \%, 12$ coverslips from three cultures) and methylglyoxal $(77.3 \pm 1.1 \%, 12$ coverslips from three cultures) treatments when analyzed by coverslip $(p=0.46$; unpaired $t$ test; $n=12$ coverslips) or by culture ( $p=0.29$; unpaired $t$ test; $n=3$ cultures; Fig. 6B). Analysis of only neurons treated with media control indicated that AIS length in CaMKII $\alpha-(27.81 \mu \mathrm{m}$; $n=298)$ was $\sim 7.5 \%$ shorter than $\mathrm{CaMKIl} \alpha+(30.08 \mu \mathrm{m}$; $n=76)$ neurons. This shorter AIS length in inhibitory neurons is consistent with a previous study of hippocampal cultures from GAD67-GFP transgenic mice (Prestigio et al., 2019). Representative images of AIS length in CaMKIl $\alpha+$ and CaMKIl $\alpha$ - neurons after $24 \mathrm{~h}$ of exposure to $100 \mu \mathrm{m}$ methylglyoxal are shown (Fig. 6C). Methylglyoxal shortened the AIS in both $\mathrm{CaMKIl} \alpha+$ and $\mathrm{CaMKIl} \alpha-$ neurons at the culture preparation level (drug: $F_{(1,8)}=24.27, p=0.001 ; n=3$ cultures). In CaMKII $\alpha+$ neurons, AIS length after methylglyoxal exposure $(26.25 \pm 0.27 \mu \mathrm{m}, n=278$ AIS from three cultures) was shorter than media control $(30.08 \pm 0.30 \mu \mathrm{m}, n=298$ AIS from three cultures) at both the AIS ( $p<0.0001$; unpaired homoscedastic two-tailed $t$ test, $12.7 \%$ reduction) and culture preparation level ( $p=0.03$; Sidak; Fig. $6 D)$. Similarly, in CaMKII $\alpha$ - neurons, AIS length after methylglyoxal exposure (23.65 $\pm 0.42 \mu \mathrm{m}, n=86$ AIS from three cultures) was shorter than media control $(27.81 \pm 0.47 \mu \mathrm{m}, n=76$ AIS from three cultures) at both the AIS ( $p<0.0001$, unpaired homoscedastic two-tailed $t$ test, $15.0 \%$ reduction) and culture preparation level $(p=0.01$; Sidak; Fig. $6 D)$. Cumulative frequency distribution of AIS length exemplifies the difference in AIS length between CaMKII $\alpha+$ and CaMKIl $\alpha$ - neurons after media control treatment as well as methylglyoxal-evoked AIS shortening in both CaMKII $\alpha+$ and CaMKII $\alpha$ - neurons (Fig. $6 E$ ).

Taken together, our results using two complementary AIS markers, Ankyring (Figs. $4 A-C, 5 C, D$ ) and $\beta$ IV spectrin (Fig. 6C-E), indicate that increase of methylglyoxal, but not insulin resistance or high glucose alone (Figs. 1, 2), could be the key initiator of AIS shortening during type 2 diabetes. Exposure to 100, but not 1 or $10, \mu \mathrm{M}$ 
A Example images
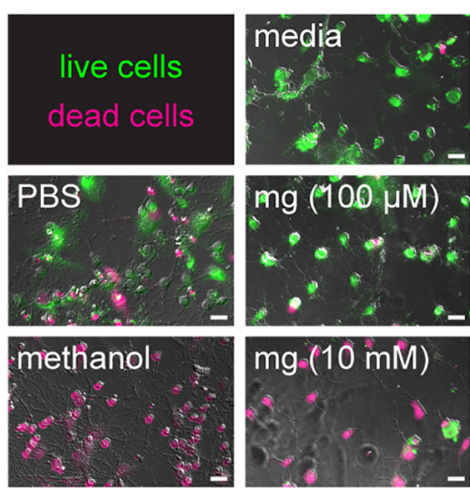

C AIS length before \& after media replacement

$\mu \mathrm{m}$

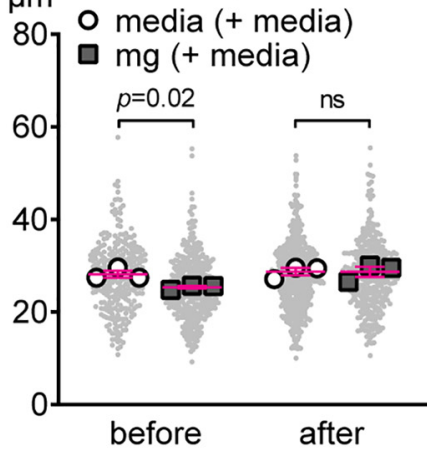

B Live/Dead cell viability

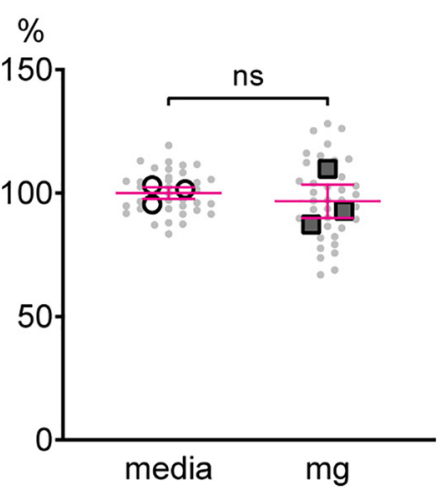

D Cumulative fraction

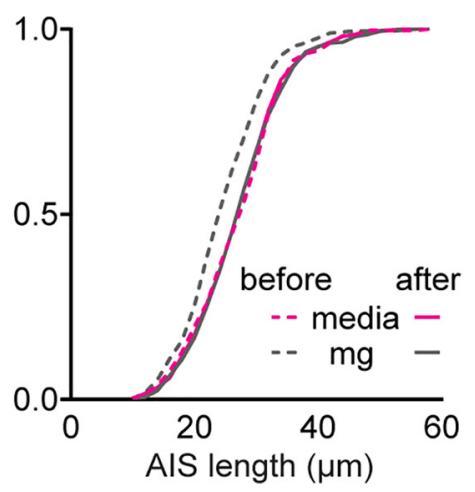

Figure 5. AIS shortening by methylglyoxal does not alter cell viability and is reversible. $\boldsymbol{A}$, Representative images of live/dead assay results after PBS (control) or methanol (to induce cell death) exposure for $15 \mathrm{~min}$ and after $24 \mathrm{~h}$ of exposure to media, $100 \mu \mathrm{m}$ methylglyoxal, or $10 \mathrm{~mm}$ methylglyoxal. Scale bar: $20 \mu \mathrm{m}$. B. Cell viability (live/dead assay shown as the \% of live cells normalized to media control) results after $24 \mathrm{~h}$ of exposure to media or $100 \mu \mathrm{m}$ methylglyoxal. Small gray dots indicate each image and larger open symbols represent the average $\%$ of live cells for each culture preparation (40 images were analyzed from $n=3$ cultures). ns (not significant) indicates statistical comparison at the culture preparation level. C, AIS length after an initial exposure to media or $100 \mu \mathrm{m}$ methylglyoxal for $24 \mathrm{~h}$ (before), followed by replacement with conditioned media and a 24-h recovery period (after). Small gray dots indicate each AIS and larger open symbols represent the average AIS length for each culture preparation (274-499 AIS were analyzed from $n=3$ cultures); $p$ value or ns (not significant) indicates statistical comparison at the culture preparation level. $\boldsymbol{D}$, Cumulative fractional distribution of AIS length before and after media replacement following the initial $24 \mathrm{~h}$ of exposure to media or $100 \mu \mathrm{m}$ methylglyoxal.

methylglyoxal for $24 \mathrm{~h}$ was sufficient to increase cellular methylglyoxal to potentially pathophysiological levels (Fig. 3) and shorten the AIS (Fig. 4) in a reversible manner without cell death (Fig. 5) in both excitatory and inhibitory cortical neuron populations (Fig. 6). These results suggest that $100 \mu \mathrm{m}$ methylglyoxal could be a pathophysiologically-relevant dose of methylglyoxal that leads to AIS shortening in our in vitro cortical culture model. We used this $100 \mu \mathrm{m}$ dose to characterize the time course of methylglyoxal-evoked changes in cellular excitability, neuronal network activity, and AIS length to better understand the relationship between AIS geometry and neuronal network operation.

\section{Effect of methylglyoxal on cortical neuron resting membrane potential and excitability parameters}

Subtle AIS shortening (a change in length as small as $4.5 \%$ ) is reported to reduce neuronal excitability at the cellular (Baalman et al., 2013; Evans et al., 2015; Vascak et al., 2017; Goethals and Brette, 2020; Jamann et al., 2021) and network (Fruscione et al., 2018; Sohn et al., 2019) levels. In the next experiments we tested the hypothesis that methylglyoxal-evoked AIS shortening would be associated with reduced neuronal function at both the cellular and network levels.

Previous studies indicate that depolarization by high $\mathrm{KCl}$ leads to rapid AIS shortening within 1-3 $\mathrm{h}$ (Evans et al., 2015; Jamann et al., 2021) and methylglyoxal (100$300 \mu \mathrm{M}$ ) depolarizes the resting membrane potential in rat somatosensory cortex slices within minutes (de Arriba et al., 2006) and in mouse DRG neurons within $3 \mathrm{~h}$ (Bierhaus et al., 2012). Taken together, these studies suggest that depolarization may play a role in AIS shortening by methylglyoxal. To determine the time course of changes in resting membrane potential in our cortical cultures, we performed whole-cell patch clamp recordings from pyramidal-like neurons after exposure to $100 \mu \mathrm{m}$ 
A

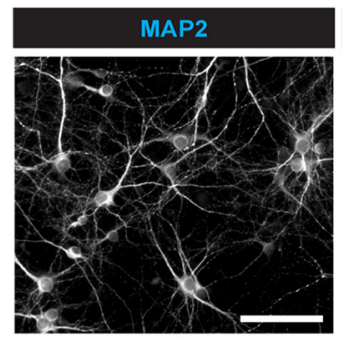

Example images of CaMKIIa+/- neuronal distribution
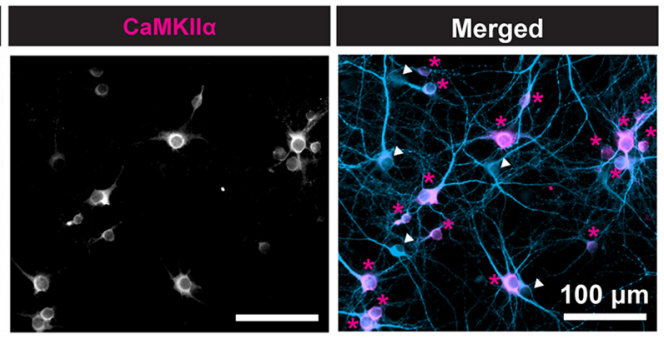

C Example images of AIS in CaMKIla+/- neurons

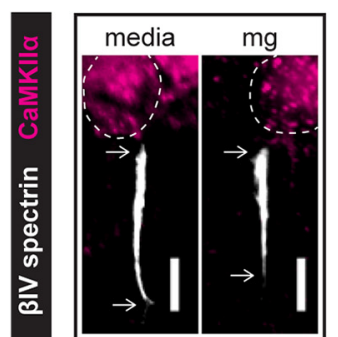

CaMKIIa+

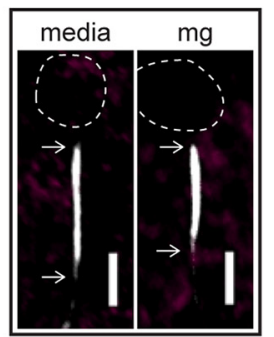

CaMKIIa-

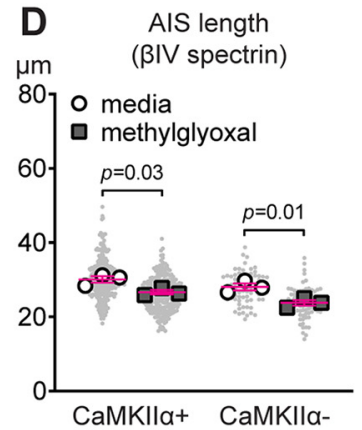

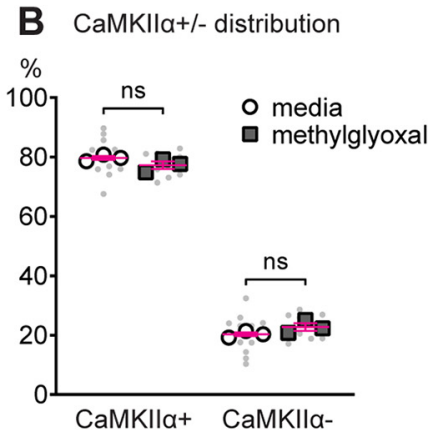

E Cumulative fraction

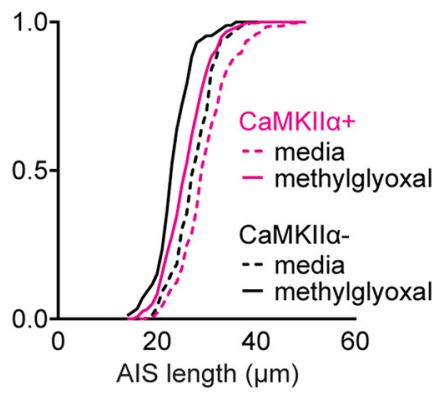

Figure 6. Methylglyoxal shortens the AIS in both excitatory and putative inhibitory neurons. A, Representative images showing MAP2 (soma and dendrites) and CaMKIl $\alpha$ (excitatory neuron marker) immunostaining in DIV14 postnatal cortical cultures. $\mathrm{CaMKIl} \alpha+$ (magenta asterisks) and CaMKIl $\alpha$ - (white arrowheads) neurons are indicated in the merged image. Scale bar: $100 \mu \mathrm{m}$. $\boldsymbol{B}$, Quantification of the percentage of total cells counted that were CaMKII $\alpha+$ (excitatory) or CaMKIl $\alpha$ - (putative inhibitory) after $24 \mathrm{~h}$ of exposure to $100 \mu \mathrm{m}$ methylglyoxal. Small gray dots indicate each coverslip and larger open symbols represent the average \% of CaMKII $\alpha+/$ - cells for each culture preparation (12 coverslips were analyzed from $n=3$ cultures). ns (not significant) indicates statistical comparison at the culture preparation level. $\boldsymbol{C}$, Representative images of the AIS ( $\beta$ IV spectrin) in CaMKII $\alpha+$ and CaMKII $\alpha-$ neurons after $24 \mathrm{~h}$ of exposure to $100 \mu \mathrm{m}$ methylglyoxal. Arrows denote the start and end of the AIS. Dashed lines indicate the neuronal soma. Scale bar: $10 \mu \mathrm{m}$. $\boldsymbol{D}$, Quantification of AIS length ( $\beta$ IV spectrin) after $24 \mathrm{~h}$ of exposure to media or $100 \mu \mathrm{m}$ methylglyoxal in $\mathrm{CaMKII} \alpha+$ and $\mathrm{CaMKII} \alpha$ - neurons. Small gray dots indicate each AIS and larger open symbols represent the average AIS length for each culture preparation (278-298 AIS were analyzed from $n=3$ cultures for CaMKIl $\alpha+, 76-86$ AIS were analyzed from $n=3$ cultures for $\mathrm{CaMKII} \alpha-) ; p$ values indicate statistical comparison at the culture preparation level. $\boldsymbol{E}$, Cumulative fractional distribution of AIS length in CaMKII $\alpha+$ and CaMKIl $\alpha$ - neurons after $24 \mathrm{~h}$ of exposure to 0-100 $\mu \mathrm{m}$ methylglyoxal.

methylglyoxal. We first recorded resting membrane potential changes in the same cell before and after continuous infusion of aCSF alone or aCSF containing $100 \mu \mathrm{m}$ methylglyoxal. Example traces of spontaneous spiking suggest that methylglyoxal may reduce action potential bursts within $0.5 \mathrm{~h}$ compared with control (Fig. $7 A$ ). Methylglyoxal caused a gradual depolarization from $-69.1 \pm 2.0 \mathrm{mV}(t=0 \mathrm{~min})$ to $-49.9 \pm 2.0(t=60 \mathrm{~min} ; n=4$ cells from four coverslips) whereas control aCSF did not change the resting membrane potential ( $n=2$ cells from 2 coverslips; Fig. $7 B$ ). This $\sim 19 \mathrm{mV}$ depolarization by methylglyoxal is similar to that reported in previous studies (de Arriba et al., 2006; Bierhaus et al., 2012). In the second set of experiments, we assessed resting membrane potential input resistance, rheobase, and voltage threshold in cultured cortical neurons during control conditions (aCSF only) or after exposure to $100 \mu \mathrm{m}$ methylglyoxal for $0.5-3$ or $24 \mathrm{~h}$ using a current injection step protocol. Resting membrane potential was depolarized by methylglyoxal (drug; $F_{(2,39)}=44.18, p<0.0001$; Tukey) at $0.5-3 \mathrm{~h}$ $(49.6 \pm 1.3 \mathrm{mV} ; n=15$ neurons from 6 coverslips) compared with control $(-69.5 \pm 1.3 \mathrm{mV} ; n=12$ neurons from 6 coverslips; $p<0.0001$ vs $0.5-3$ h) but returned to normal at $24 \mathrm{~h}(-66.3 \pm 2.0 \mathrm{mV} ; n=15$ neurons from 6 coverslips; $p=0.36$ vs control; Fig. $7 C$ ). Example traces of the step current protocol are consistent with depolarization-induced action potential blockade (Fig. 7D). Methylglyoxal reduced the input resistance (drug; $F_{(2,39)}=9.19, p=0.0005$; Tukey) at $0.5-3 \mathrm{~h}(252.7 \pm 14$ $\mathrm{M} \Omega ; p=0.002$ vs control) and at $24 \mathrm{~h}(249.0 \pm 9.9 \mathrm{M} \Omega$; $p=0.001$ vs control) compared with the control (332 $\pm 21 \mathrm{M} \Omega$; Fig. 7E). Threshold current necessary to trigger an action potential (rheobase) was reduced by methylglyoxal (drug; $F_{(2,30)}=7.66, p=0.002$; Tukey) at $0.5-3$ h $(60.2 \pm 3.1 \mathrm{pA} ; n=6$ cells from 6 coverslips; $p=0.007$ vs control) compared with the control (105.8 $\pm 9.1 \mathrm{pA} ; n=12$ cells from 6 coverslips) and returned to normal at $24 \mathrm{~h}(110.5 \pm 7.4 \mathrm{pA} ; n=15$ cells from 6 coverslips; $p=0.89$ vs control; Fig. 7F). Methylglyoxal slightly altered the threshold potential (drug; $F_{(2,30)}=4.41$, $p=0.02$; Tukey) with a small but non-significant increase at $0.5-3 \mathrm{~h}(-34.5 \pm 1.6 \mathrm{mV} ; n=6$ cells from 6 coverslips; $p=0.37$ vs control) and a slight reduction at $24 \mathrm{~h}(-42.0 \pm 1.4 \mathrm{mV} ; n=15$ cells from 6 coverslips; $p=0.02$ vs $0.5-3$ h) compared with the control 

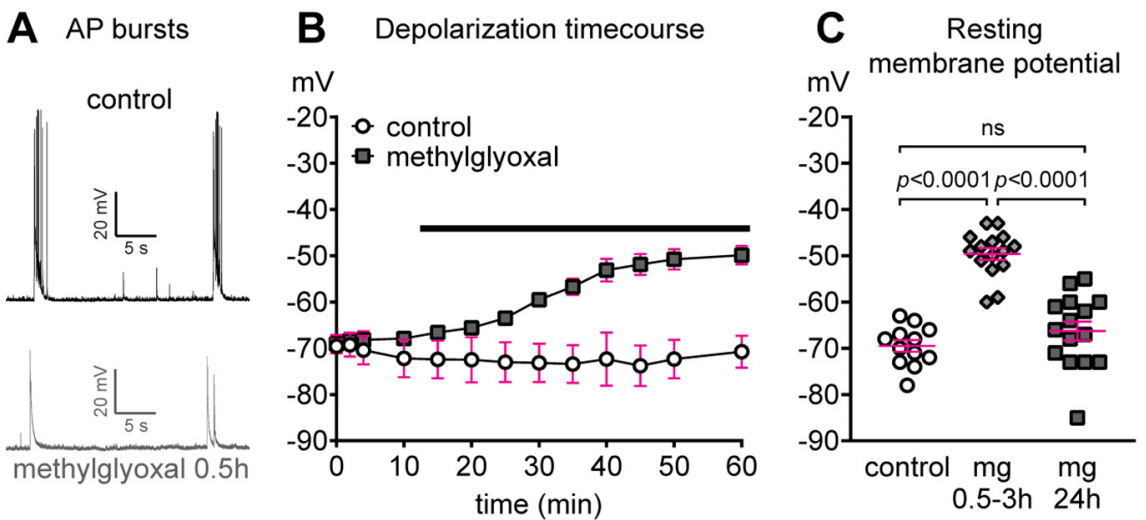

D Example voltage traces after current injection step protocol

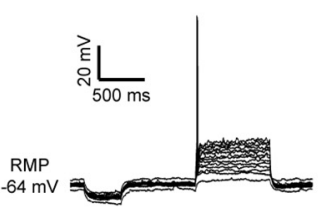

control

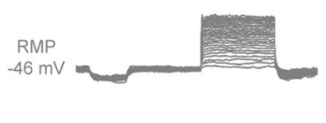

methylglyoxal $0.5 \mathrm{~h}$

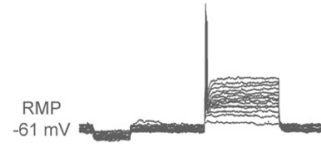

methylglyoxal $24 \mathrm{~h}$
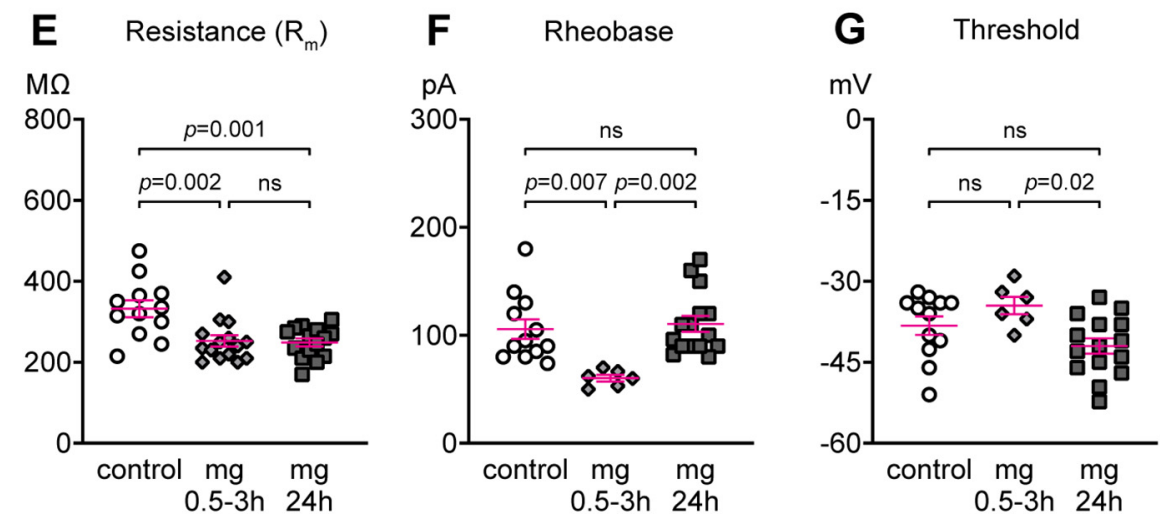

Figure 7. Depolarization of resting membrane potential by methylglyoxal. A, Example traces of spontaneous action potentials (APs) in aCSF control (top) or $0.5 \mathrm{~h}$ after exposure to $100 \mu \mathrm{m}$ methylglyoxal (bottom). $\boldsymbol{B}$, Time course of changes in resting membrane potential during exposure to aCSF control ( $n=2$ cells) or $100 \mu \mathrm{m}$ methylglyoxal ( $n=4$ cells) conditions. Black bar indicates continuous infusion of aCSF only or aCSF containing $100 \mu \mathrm{m}$ methylglyoxal. C, Quantification of resting membrane potential in aCSF control (control; $n=12$ cells from 6 coverslips) or at $0.5-3 \mathrm{~h}$ ( $n=15$ cells from 6 coverslips) or $24 \mathrm{~h}$ ( $n=15$ cells from 6 coverslips) after exposure to $100 \mu \mathrm{m}$ methylglyoxal. Depolarization of resting membrane potential by methylglyoxal at $0.5-3 \mathrm{~h}$ recovered by $24 \mathrm{~h}$. $\boldsymbol{D}$, Representative voltage traces during the current injection step protocol (500-ms duration, $10 \mathrm{pA}$ per step) in aCSF control (14 steps) or at $0.5 \mathrm{~h}$ (27 steps) or $24 \mathrm{~h}$ (14 steps) after exposure to $100 \mu \mathrm{m}$ methylglyoxal. The voltage traces are plotted on the same $y$-axis (data not shown), and the resting membrane potential for each cell is indicated. $\boldsymbol{E}$, Resistance of the membrane $\left(\mathrm{R}_{\mathrm{m}}\right)$ in aCSF control or at $0.5-3 \mathrm{~h}$ or $24 \mathrm{~h}$ after exposure to $100 \mu \mathrm{m}$ methylglyoxal. $\boldsymbol{F}$, Rheobase (current threshold) in aCSF control or at $0.5-3 \mathrm{~h}$ or $24 \mathrm{~h}$ after exposure to $100 \mu \mathrm{m}$ methylglyoxal. Note that only 6 out of 15 cells were able to fire APs at $0.5-3 \mathrm{~h}$, whereas 15 out of 15 cells fired APs at $24 \mathrm{~h}$. G, Threshold potential in aCSF control or at $0.5-3 \mathrm{~h}$ or $24 \mathrm{~h}$ after exposure to $100 \mu \mathrm{m}$ methylglyoxal. Note that only 6 out of 15 cells were able to fire APs at $0.5-3 \mathrm{~h}$ whereas 15 out of 15 cells fired APs at 24 h. $\boldsymbol{C}, \boldsymbol{E}, \boldsymbol{F}, \boldsymbol{G}$ : $p$ value or ns (not significant) indicates statistical comparison at the cellular level.

$(-38.2 \pm 1.7 \mathrm{mV} ; n=12$ cells from 6 coverslips; $p=0.19$ vs $24 \mathrm{~h}$; Fig. 7G). Importantly, 7 out of 15 cells at $0.5-3 \mathrm{~h}$ were unable to fire even with up to 260 pA current injection, possibly because depolarization of resting membrane potential caused inactivation of sodium channels.

\section{Effect of methylglyoxal on neuronal network activity}

Our results (Fig. 7) and previous studies indicate that methylglyoxal acutely alters neuronal activity at the cellular level (de Arriba et al., 2006; Bierhaus et al., 2012; Radu et al., 2012; Andersson et al., 2013; Griggs et al., 2019). To determine the effect of methylglyoxal on the coordinated activity of neuronal networks we used MEA recordings of our cortical cultures. Characterization of MEA network activity before drug exposure revealed that MEAwide network spike frequency increased from DIV7 $(44.5 \pm 4.8 \mathrm{~Hz})$ to DIV13 $(230.6 \pm 10.9 \mathrm{~Hz} ; p<0.0001$; $n=9$ MEAs from three cultures). Example sixty second 
A

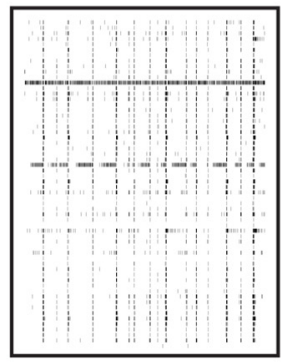

$-1$
Example raster plots (60 s duration)

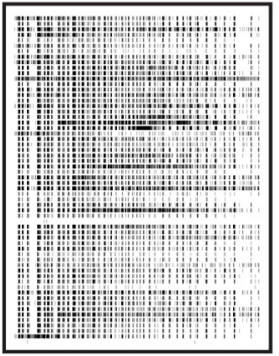

1

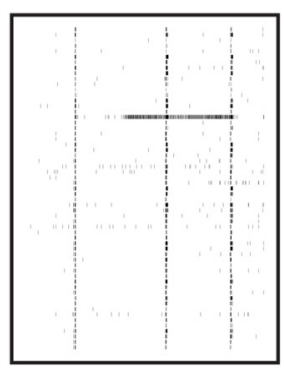

3

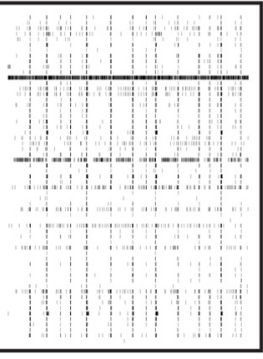

15

Minutes after adding $100 \mu \mathrm{M}$ methylglyoxal
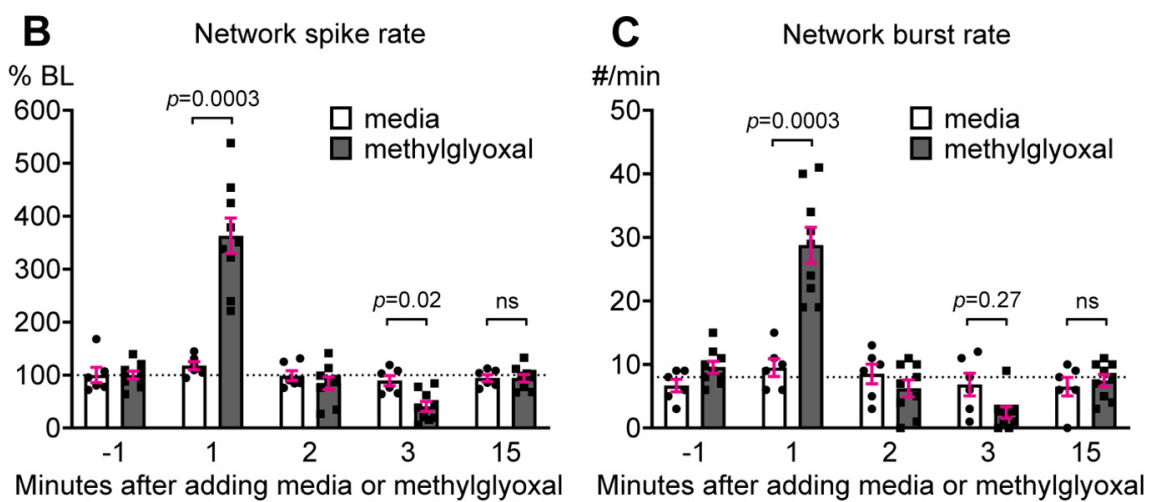

Figure 8. Methylglyoxal transiently increases neuronal network activity within the first few minutes. $\boldsymbol{A}$, Representative raster plots (60-s duration) of MEA recordings showing spike activity (60 electrodes, one per row) during 1-min intervals before and after exposure to $100 \mu \mathrm{m}$ methylglyoxal. B, Quantification of network spike rate (normalized as percent of -1-min baseline) during 1-min intervals before and after exposure to media or $100 \mu \mathrm{M}$ methylglyoxal $(n=6$ MEAs from 2 cultures for media and $n=9$ MEAs from 3 cultures for methylglyoxal). Dashed line indicates baseline. C, Quantification of network burst rate (MEA-wide bursts per minute) during 1-min intervals before and after exposure to media or $100 \mu \mathrm{M}$ methylglyoxal $(n=6$ MEAs from 2 cultures for media and $n=9$ MEAs from 3 cultures for methylglyoxal). Dashed line indicates baseline.

raster plots from DIV13 show that adding $100 \mu \mathrm{m}$ methylglyoxal resulted in an immediate increase of network activity, followed by a short period of quiescence, and restoration to baseline activity within $15 \mathrm{~min}$ (Fig. 8A). Methylglyoxal altered network spike rate compared with media control (drug $\times$ time interaction: $F_{(4,52)}=31.16$; $p<0.0001 ; n=6$ MEAs from 2 cultures for media control and $n=9$ MEAs from three cultures for methylglyoxal; Sidak; Fig. 8B). Methylglyoxal increased network spike rate $(363.0 \pm 33.5 \%)$ compared with media control $(117.7 \pm 7.5 \%)$ during the first minute by $\sim 300 \%$. At $3 \mathrm{~min}$, methylglyoxal reduced network spike rate $(40.9 \pm$ $9.5 \%)$ to $\sim 50 \%$ of media control $(89.3 \pm 9.6 \%)$. At $15 \mathrm{~min}$, network spike rates were similar to baseline after either methylglyoxal $(p=0.99)$ or media $(p=0.73)$ exposure. Similarly, methylglyoxal increased the network burst rate (number of MEA-wide network bursts per minute) immediately after its addition with restoration of baseline bursting at $15 \mathrm{~min}$ (drug $\times$ time interaction: $F_{(4,52)}=24.45 ; p<0.0001$; Sidak; Fig. $8 C$ ).

We also recorded from the same MEAs at later timepoints to examine how cellular depolarization at $0.5-3 \mathrm{~h}$ (Fig. 7C) or AIS shortening at $24 \mathrm{~h}$ (Figs. 4-6) affect neuronal network activity. Example raster plots of neuronal network activity are shown at baseline and at 3 and $24 \mathrm{~h}$ after exposure to $100 \mu \mathrm{M}$ methylglyoxal (Fig. 9A). Control exposure to media alone did not alter network spike rate (repeated measures across time: $F_{(3,15)}=1.92, p=0.17$; $n=6$ MEAs from 2 cultures; Fig. 9B). Methylglyoxal altered network spike rate (repeated measures across time: $F_{(3,24)}=18.9, p<0.0001 ; n=9$ MEAs from three cultures), with reduced spiking at 0.5 and $3 \mathrm{~h}$ that returned to baseline level at $24 \mathrm{~h}$ (Fig. 9C). Media control did not alter network burst spikes (number of spikes within network bursts; Fig. $9 D$ ) or network burst duration (Fig. 9E). Network burst rate (number of network bursts per min) in media control MEAs slightly increased during the experiment (Fig. 9F). Network burst spike rate (number of spikes per second within a network burst) was lower at $3 \mathrm{~h}$ and unchanged at $24 \mathrm{~h}$ by media control treatment (Fig. 9G). Methylglyoxal altered network burst spikes (Fig. $9 H$ ) and network burst duration (Fig. 9/) at $3 \mathrm{~h}$ without changing the network burst rate (Fig. 9J) or network burst spike rate (Fig. 9K).

\section{Spiking activity is not required for methylglyoxal- evoked AIS shortening}

Our results indicate that reduction of network activity by methylglyoxal at $0.5-3 \mathrm{~h}$ returned to normal at $24 \mathrm{~h}$ (Fig. 9C) when we observed a shorter AIS (Figs. 4-6). This 
A

Example raster plots (30 s duration)

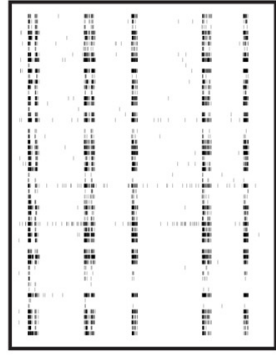

Baseline (BL)

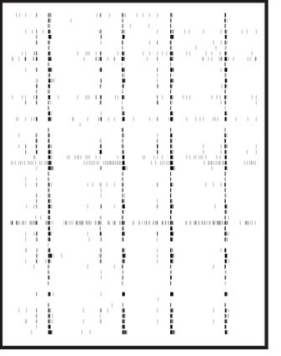

$3 \mathrm{~h}$ after adding $\mathrm{mg}$

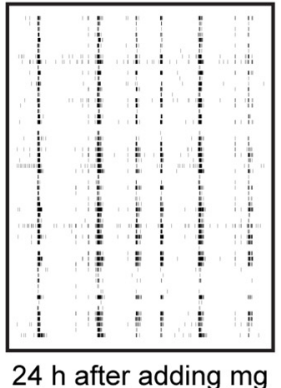

$24 \mathrm{~h}$ after adding $\mathrm{mg}$

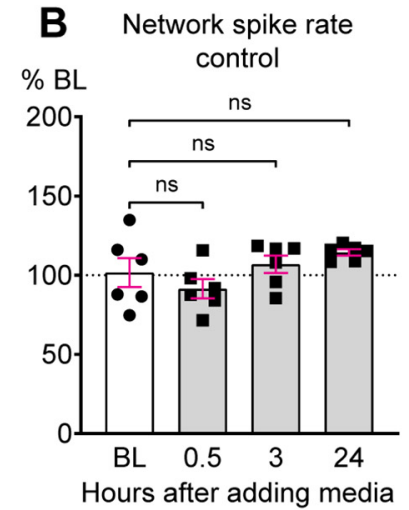

F Network burst rate control

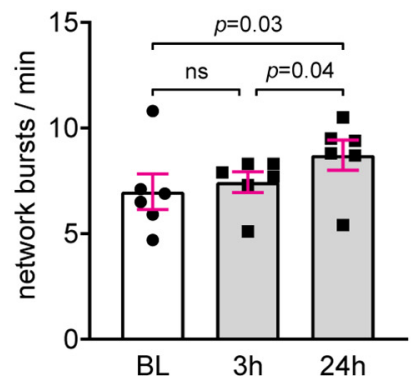

J

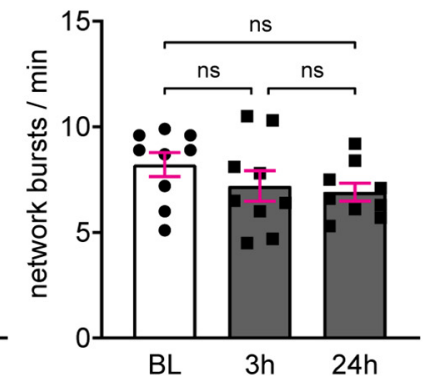

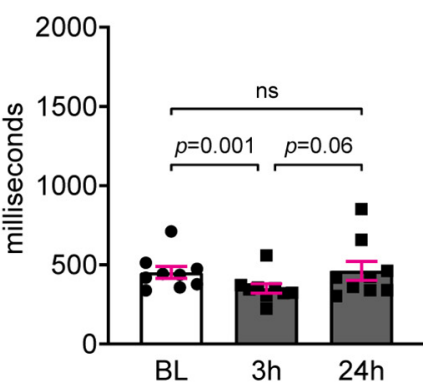

H $\begin{gathered}\text { Network burst spikes } \\ \text { methylglyoxal }\end{gathered}$

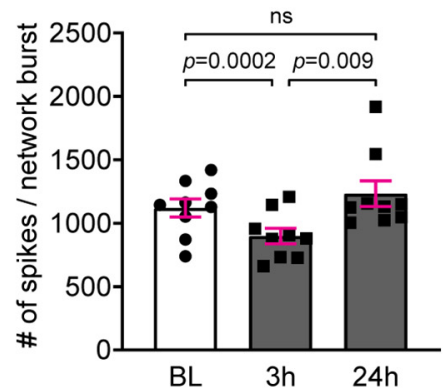

$24 \mathrm{~h}$ etwork burst duratio

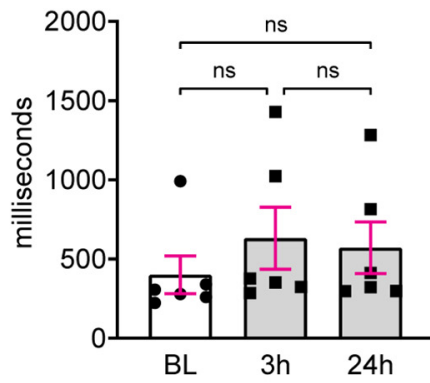

I

E Network burst duration control

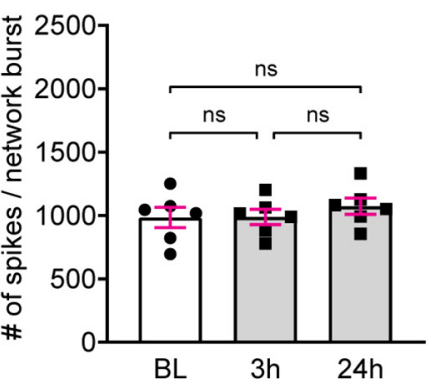

H Network burst spikes

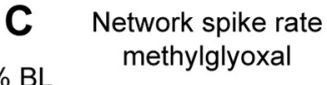

$\% \mathrm{BL}$

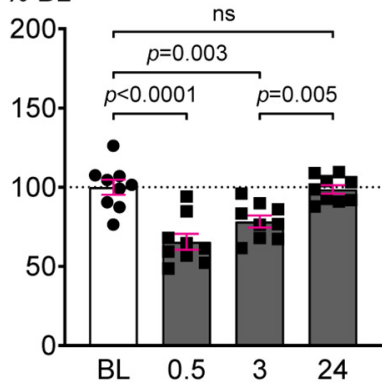

Hours after adding $\mathrm{mg}$
G Network burst spike rate control

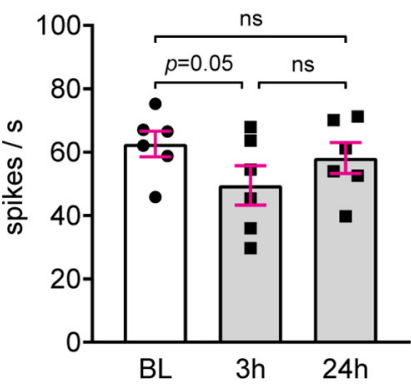

K Network burst spike rate methylglyoxal

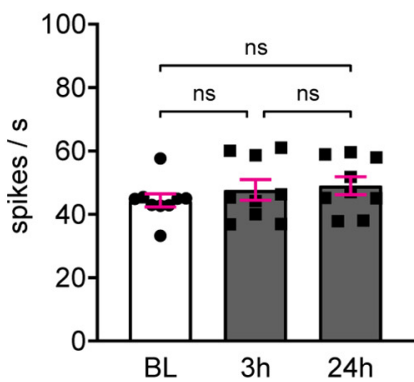

Figure 9. Reduction of neuronal network activity by methylglyoxal at $0.5-3 \mathrm{~h}$ recovers by $24 \mathrm{~h}$. $\boldsymbol{A}$, Representative raster plots (30-s duration) showing spike activity (60 electrodes, one per row) during 15-min MEA recordings at baseline (BL) and 3 and $24 \mathrm{~h}$ after exposure to $100 \mu \mathrm{m}$ methylglyoxal. $\boldsymbol{B}$, Network spike rate after media control exposure ( $n=6$ MEAs from 2 cultures). $\boldsymbol{C}$, Network spike rate after methylglyoxal exposure ( $n=9$ MEAs from 3 cultures). $\boldsymbol{D}-\mathbf{G}$, Network spiking parameters after media control exposure. $\boldsymbol{H}-\boldsymbol{K}$, Network spiking parameters after methylglyoxal exposure. $\boldsymbol{B}-\boldsymbol{K}$ : $\boldsymbol{p}$ values or ns (not significant) indicate statistical comparison at the MEA level.

might suggest that AIS shortening is a homeostatic response to transient changes (increase at 1 min followed by decrease at $0.5-3 \mathrm{~h}$ ) in network spiking and bursting. However, previous studies of AIS plasticity indicate that action potential spiking is not required for high $\mathrm{KCl}$ evoked relocation (Grubb and Burrone, 2010) or shortening (Evans et al., 2015). To test whether methylglyoxalevoked AIS shortening depends on the biphasic changes in spiking activity uncovered by MEA recordings (Figs. 8, 9), we co-exposed our cortical cultures to $100 \mu \mathrm{m}$ methylglyoxal plus the sodium channel blocker TTX (1 $\mu \mathrm{M})$ for 24 $\mathrm{h}$ and assessed spiking activity via MEAs and AIS length via AnkyrinG immunostaining. Example $60 \mathrm{~s}$ duration rasterplots (Fig. 10A) and quantification of network spike frequency ( $n=1$ MEA; Fig. 10B) show spiking and network bursting at baseline are almost completely abolished within seconds of adding $1 \mu \mathrm{M}$ TTX whereas network activity after addition of water control is relatively unchanged. Elimination of network activity by TTX persisted for at least $24 \mathrm{~h}$, when AIS length was quantified. Representative images after co-exposure to methylglyoxal and TTX are shown (Fig. 10C). Compared with media control, methylglyoxal shortened the AIS when co-exposed with water or TTX at both the AIS $(p<0.0001$; unpaired homoscedastic $t$ test; $n=295-335$ AIS from three cultures) and culture preparation level (main effect of methylglyoxal; $F_{(1,8)}=110.4, p<0.0001 ; n=3$ cultures; Fig. $\left.10 D\right)$. Importantly, AIS length was similar after co-exposure to 
A Example rasterplots (60 s duration)

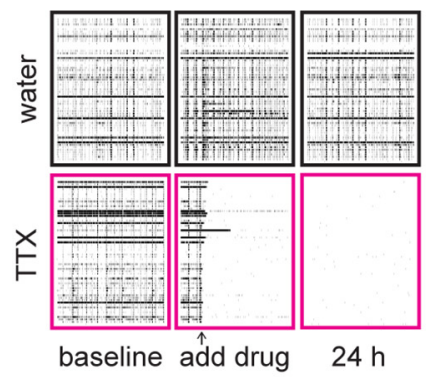

B Network spike frequency $\% \mathrm{BL}$

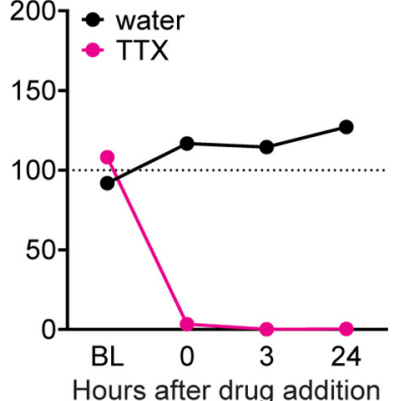

C

Example images of the AIS (AnkyrinG)

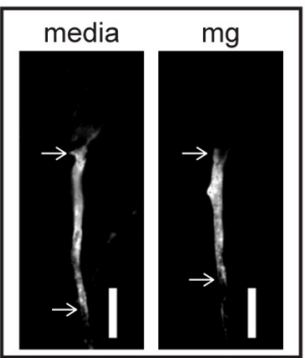

water

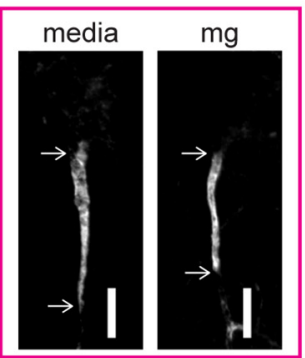

TTX

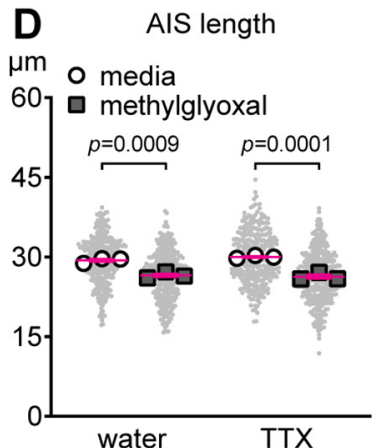

E Cumulative frequency

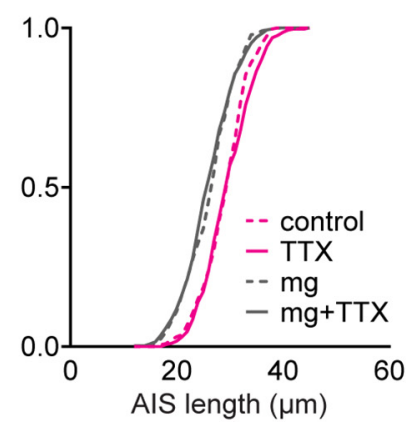

Figure 10. Methylglyoxal-evoked AIS shortening is independent of spiking activity. $\boldsymbol{A}$, Representative raster plots (60-s duration) showing spike activity (60 electrodes, one per row) at baseline, after adding drug (water or $1 \mu \mathrm{M} T \mathrm{TX}$ ), and at $24 \mathrm{~h}$. B, Quantification of network spike rate (normalized as percent of baseline) before (BL) and after exposure to water or $1 \mu \mathrm{M}$ TTX $(n=1$ MEA). Dashed line indicates baseline average. $\boldsymbol{C}$, Representative images of Ankyring (AIS) immunostaining $24 \mathrm{~h}$ after exposure to media or $100 \mu \mathrm{m}$ methylglyoxal alone or in combination with water or $1 \mu \mathrm{m}$ TTX. Scale bar: $10 \mu \mathrm{m}$. D, AIS length $24 \mathrm{~h}$ after co-exposure to media or methylglyoxal plus water or TTX indicating that TTX does not alter methylglyoxal-evoked AIS shortening. Small gray dots indicate each AIS and larger open symbols represent the average AIS length for each culture preparation (295-335 AIS were analyzed from $n=3$ cultures); $p$ values indicate statistical comparison at the culture preparation level. $\boldsymbol{E}$, Cumulative fractional distribution of AIS length after $24 \mathrm{~h}$ of exposure to methylglyoxal+TTX.
A Example immunofluorescence images (AnkyrinG)
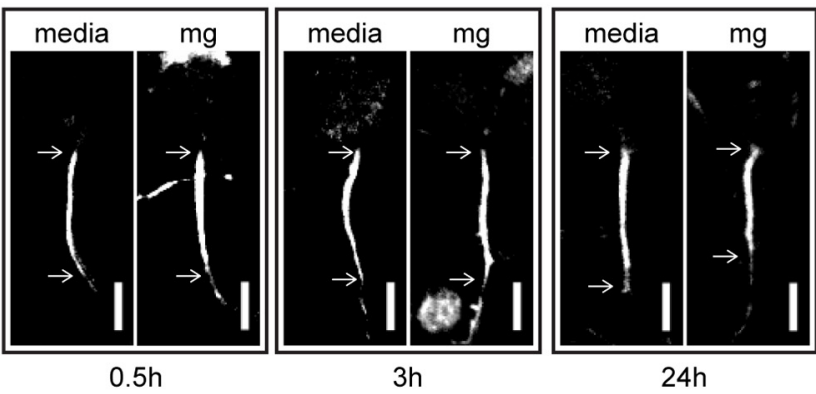

B

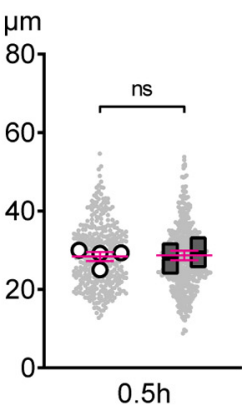

C

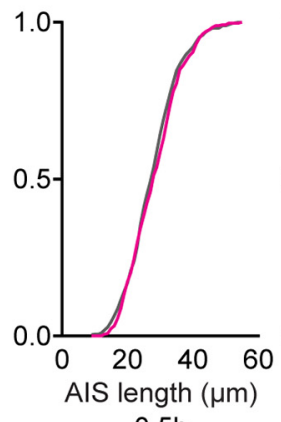

$0.5 \mathrm{~h}$

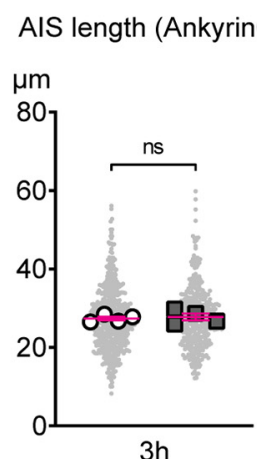

$3 \mathrm{~h}$

Cumulative fraction

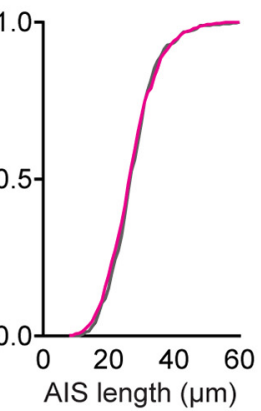

$3 \mathrm{~h}$
O media

methylglyoxal

$\mu \mathrm{m}$

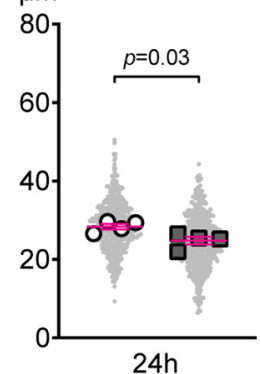

$24 h$

- media - methylglyoxa

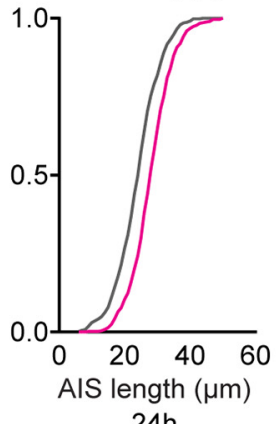

$24 \mathrm{~h}$
Figure 11. Time course of AIS shortening by methylglyoxal. $\boldsymbol{A}$, Representative images of AnkyrinG (AIS) immunostaining at 0.5, $3,24 \mathrm{~h}$ after media or $100 \mu \mathrm{m}$ methylglyoxal exposure. Scale bar: $10 \mu \mathrm{m}$. $\boldsymbol{B}$, AIS length at $0.5,3,24 \mathrm{~h}$ after media control treatment indicating AIS shortening at $24 \mathrm{~h}$ but not 0.5 or $3 \mathrm{~h}$. Small gray dots indicate each AIS and larger open symbols represent the average AIS length for each culture preparation (377594 AIS were analyzed from $n=4$ cultures); $p$ values or ns (not significant) indicate statistical comparison at the culture preparation level. $\mathbf{C}$, Cumulative fractional distribution of AIS length after $0.5,3$, and $24 \mathrm{~h}$ of exposure to methylglyoxal.

media + water (control) or media+TTX ( $p=0.49$; Tukey; $n=3$ cultures) and after co-exposure to methylglyoxal + water or methylglyoxal+TTX $(p=0.93$; Tukey; $n=3$ cultures). Cumulative frequency distribution of AIS length exemplifies the methylglyoxal-evoked AIS shortening in the presence or absence of TTX (Fig. 10E). These results indicate that neuronal spiking (action potentials) is not required for methylglyoxal-evoked AIS shortening, even though methylglyoxal temporarily changes network 
activity (Figs. 8,9). In addition, they show that almost complete elimination of spontaneous network activity by TTX alone does not change AIS length at $24 \mathrm{~h}$.

\section{Time course of AIS shortening by methylglyoxal}

Our findings at $24 \mathrm{~h}$ after methylglyoxal exposure (Figs. $4,7,9)$ refute our initial hypothesis that AIS shortening would coincide with neuronal depolarization and reduced network activity. However, since we observed depolarization and reduced network activity at 0.5-3 h after methylglyoxal exposure (Figs. 7,9), and depolarization by high $\mathrm{KCl}$ leads to AIS shortening as rapid as $1 \mathrm{~h}$ in somatosensory cortex slices (Jamann et al., 2021) and $3 \mathrm{~h}$ in hippocampal neuron cultures (Evans et al., 2015), we checked AIS length at earlier timepoints. Representative images of AIS length after $0.5,3$, and $24 \mathrm{~h}$ of exposure to media or methylglyoxal are shown (Fig. 11A). AIS length at $0.5 \mathrm{~h}$ was similar when analyzed at both the AIS $(p=0.20$; unpaired homoscedastic $t$ test; $n=377-530$ AIS from four cultures) and culture preparation $(p=0.86$; unpaired homoscedastic $t$ test; $n=4)$ levels (Fig. 11B). AIS length at $3 \mathrm{~h}$ was also similar at both the AIS ( $p=0.38$; unpaired homoscedastic $t$ test; $n=397-$ 556 from four cultures) and culture preparation $(p=0.70$; unpaired homoscedastic $t$ test; $n=4)$ levels (Fig. 11B). At $24 \mathrm{~h}$, AIS length was reduced at both the AIS ( $p<0.0001$; unpaired homoscedastic $t$ test; $n=594-531$ AIS from four cultures) and culture preparation ( $p=0.03$; unpaired homoscedastic $t$ test; $n=4$ ) levels (Fig. 11B), confirming our previous results (Figs. 4-6). Cumulative frequency distributions illustrate methylglyoxal-evoked AIS shortening at $24 \mathrm{~h}$ but not at 0.5 or $3 \mathrm{~h}$ (Fig. $11 \mathrm{C}$ ).

Contrary to AIS shortening within $1-3 \mathrm{~h}$ by high $\mathrm{KCl}$ (Evans et al., 2015; Jamann et al., 2021), we did not observe AIS shortening within $0.5-3 \mathrm{~h}$ of exposure to $100 \mu \mathrm{M}$ methylglyoxal (Fig. 11), although methylglyoxal depolarized the neurons within this short 0.5 to $3 \mathrm{~h}$ time frame (Fig. 7B,C). Instead, we observed that AIS shortening by methylglyoxal was associated with normalization of resting membrane potential (Fig. 7C) and MEA network spiking (Fig. 9C) at $24 \mathrm{~h}$. These observations, along with AIS shortening in both $\mathrm{CaMKII} \alpha+$ and $\mathrm{CaMKII} \alpha$ - neurons (Fig. 6), hint that AIS shortening by methylglyoxal is likely different from homeostatic, cell type-dependent AIS shortening after depolarization by $\mathrm{KCl}$.

\section{Discussion}

\section{Methylglyoxal mediates AIS shortening}

Because of the association between AIS shortening and cognitive impairment in type 2 diabetes we sought to identify the initiator of AIS shortening. Canonical features of type 2 diabetes, insulin resistance (Fig. 1) or high glucose (Fig. 2), did not dramatically alter AIS length in mouse cortical neuron cultures. In contrast, increase of methylglyoxal $(100 \mu \mathrm{m})$ produced $11.5 \%$ AIS shortening (Fig. 4) that was recoverable and not associated with cell death (Fig. 5). This is consistent with $8-16 \%$ AIS shortening in the prefrontal cortex of type 2 diabetic $\mathrm{db} / \mathrm{db}$ mice at 10 weeks of age with lack of apoptotic cell death (Yermakov et al., 2018). Methylglyoxal elevation leads to accumulation of MG$\mathrm{H} 1$ in patients with type 2 diabetes (Schalkwijk and Stehouwer, 2020) and both methylglyoxal (Bierhaus et al., 2012) and MG-H1 (Griggs et al., 2019) are elevated in $\mathrm{db} / \mathrm{db}$ mice that also display AIS shortening and cognitive impairment (Yermakov et al., 2018, 2019). Adding $100 \mu \mathrm{m}$ methylglyoxal to culture media increased MG-H1 in vitro (Fig. 3), indicating that our culture model recapitulates pathophysiological disruption of methylglyoxal metabolism that might be relevant to diabetes. These results suggest that methylglyoxal is a key mediator of AIS shortening in the type 2 diabetic brain. However, we recognize the reductionist in vitro approach herein cannot completely recapitulate chronic diabetic conditions in vivo. We based our study on the limited information available in the literature suggesting 1-40 $\mu \mathrm{m}$ is the normal, physiological range of methylglyoxal in CNS tissues (Kuhla et al., 2005; Kurz et al., 2011; Distler et al., 2012; Eberhardt et al., 2012; Rabbani and Thornalley, 2014; Liu et al., 2017). Since cellular levels of methylglyoxal in the brain during neurological disease are not established, it is possible that the $100 \mu \mathrm{m}$ methylglyoxal required to increase cellular MG-H1 and produce AIS shortening in the current in vitro study does not represent pathophysiological levels in vivo. Nevertheless, it is reasonable to think that prolonged increase of methylglyoxal, or the combined disruption of methylglyoxal, insulin, and glucose metabolism, during type 2 diabetes could result in sustained AIS shortening and CNS dysfunction.

\section{Methylglyoxal alters neuronal function at the cellular and network levels}

Previous studies indicate that concentrations of methylglyoxal $>1 \mathrm{~mm}$ were required to generate rapid inward currents in TRPA1-expressing HEK293 cells (Eberhardt et al., 2012) and mobilize calcium in DRG (Eberhardt et al., 2012) or spinal cord dorsal horn (Griggs et al., 2019) neurons. We observed that a much lower dose of $100 \mu \mathrm{m}$ methylglyoxal rapidly changed both cellular and network function in our cortical cultures. At the network level, MEA spiking and bursting were increased for $\sim 1 \mathrm{~min}$ followed by a quick recovery to baseline within $15 \mathrm{~min}$ (Fig. 8). At the single-cell level, depolarization of resting membrane potential occurred within minutes, persisted for at least $3 \mathrm{~h}$, and was associated with decreased rheobase and input resistance (Fig. 7). This is consistent with depolarization by $300 \mu \mathrm{m}$ methylglyoxal in neocortical somatosensory neurons within minutes (de Arriba et al., 2006) or depolarization and decrease of rheobase in DRG neurons by $100 \mu \mathrm{m}$ methylglyoxal exposure for $3 \mathrm{~h}$ (Bierhaus et al., 2012). The depolarization in our study (Fig. 7) coincided with a reduction of network spike rate and number of spikes per network burst (Fig. 9), suggesting that decreased network activity by methylglyoxal at $3 \mathrm{~h}$ might be because of depolarizing block of sodium channels. Interestingly, except for input resistance, these changes 
in cellular and network function recovered by $24 \mathrm{~h}$ despite the presence of a shorter AIS.

\section{How does methylglyoxal trigger AIS shortening and alter neuronal function?}

The detailed mechanisms underlying methylglyoxalevoked changes in network operation and AIS geometry in the current study remain unknown. Depolarization might contribute to the reduction of network activity at $0.5-3 \mathrm{~h}$ in addition to its potential role in AIS shortening (discussed below). Other explanations for altered neuronal function by methylglyoxal include decreased glutamate uptake (de Arriba et al., 2006; Lissner et al., 2021), depletion of mitochondrial ATP production (de Arriba et al., 2006, 2007), changes in the biophysical properties of sodium channels (Bierhaus et al., 2012), the cation channel transient receptor potential ankyrin A (TRPA1; Eberhardt et al., 2012; Andersson et al., 2013), or $\mathrm{GABA}_{\mathrm{A}}$ receptors (Distler et al., 2012). Potential intracellular effectors of methylglyoxal-evoked AIS shortening include calcium, calpain, calcineurin, or tau. Methylglyoxal promotes calcium mobilization in neurons (Radu et al., 2012; Griggs et al., 2019), activation of calpain (Griggs et al., 2018) and calcineurin (Maeta et al., 2005), and hyperphosphorylation of tau (Li et al., 2012). Likewise, calcium channels (Grubb and Burrone, 2010; Evans et al., 2013, 2015; Chand et al., 2015), calpain (Schafer et al., 2009; Clark et al., 2017), calcineurin (Evans et al., 2015), and tau (Sohn et al., 2019) are all implicated in AIS disruption. Another intriguing candidate is hyperpolarization-activated cyclic nucleotide-gated ( $\mathrm{HCN})$ channels. Methylglyoxal increases HCN channel current (Shimatani et al., 2015) and activation of HCN channels specifically within the AIS is reported to decrease spike probability (Ko et al., 2016). This could explain the decreased spiking activity at $0.5-3 \mathrm{~h}$ (Fig. 9). Future studies are needed to determine whether the mechanisms described above are involved in the changes in neuronal function and AIS geometry that occur when methylglyoxal metabolism is disrupted.

\section{Are AIS geometry and neuronal function linked?}

Several previous studies informed our initial hypothesis that AIS shortening would lead to reduced single-cell excitability and neuronal network activity. At the single-cell level, AIS shortening dampens multiple spike firing and increases firing threshold in vitro (Evans et al., 2015) and in vivo (Jamann et al., 2021). In silico, AIS shortening is associated with increase of interspike interval (Baalman et al., 2013), voltage threshold (Baalman et al., 2013; Goethals and Brette, 2020), and action potential acceleration (Vascak et al., 2017) in single neurons. These findings at the cellular level are consistent with shorter AIS length and reduced MEA burst rate at the network level (Fruscione et al., 2018; Sohn et al., 2019). By contrast, we observed depolarization of resting membrane potential, decreased rheobase, increased voltage threshold (Fig. 7) and reduced network spike and burst rates (Fig. 9) were not associated with shorter AIS length at
$3 \mathrm{~h}$ (Fig. 11). Instead, the AIS was shorter at $24 \mathrm{~h}$ when single-cell excitability parameters and network activity recovered to baseline levels. These surprising findings might be explained by studies showing that AIS shortening does not reduce excitability when associated with changes in Nav phosphorylation (Evans et al., 2015), loss of Kv1 channels (Sanders et al., 2020), or alteration of action potential backpropagation (Radecki et al., 2018). Determining how simultaneous AIS shortening in multiple cell types and recovery of neuronal function are related will be critical going forward.

Differences in the pattern of AIS shortening and altered neuronal function elicited by methylglyoxal versus $\mathrm{KCl}$ highlight the importance of determining how the AIS and neuronal network operation are altered by pathophysiologically-relevant factors. AIS shortening has been described as a homeostatic mechanism that reduces neuronal excitability in response to depolarization by elevating $\mathrm{KCl}$ (Evans et al., 2015). In contrast, we did not observe concurrent AIS shortening (Fig. 11) and reduced network activity (Fig. 9). It is tempting to conclude that AIS shortening at $24 \mathrm{~h}$ is a homeostatic response to the transient changes in network activity (Figs. 8,9). However, methylglyoxal-evoked AIS shortening occurred even when action potentials were blocked by TTX (Fig. 10). Moreover, addition of TTX alone blocked network activity without changing AIS length (Fig. 10). These results are consistent with the idea that AIS plasticity is independent from network spiking activity (Grubb and Burrone, 2010; Evans et al., 2015). Depolarization is suggested to be a key factor in the rapid AIS shortening induced by high $\mathrm{KCl}$ at $3 \mathrm{~h}$ (Evans et al., 2015). Depolarization may contribute to AIS shortening in the current results, since it preceded AIS shortening, but the temporal relationship of depolarization and AIS shortening by methylglyoxal is different compared with increase of $\mathrm{KCl}$. Although resting membrane potential was depolarized at $0.5-3 \mathrm{~h}$ (Fig. 7), we did not observe rapid AIS shortening by methylglyoxal at $0.5-3 \mathrm{~h}$ (Fig. 11). Instead, we observed AIS shortening at $24 \mathrm{~h}$ (Fig. 11) when resting membrane potential recovered to normal (Fig. 7). Another difference in the pattern of AIS shortening by methylglyoxal compared with $\mathrm{KCl}$ is the cell types involved. Evans et al. (2015) described celltype specific AIS shortening in excitatory CA3 but not inhibitory GABAergic hippocampal neurons after $3 \mathrm{~h}$ of exposure to $\mathrm{KCl}$. We observed cell-type independent AIS shortening in both excitatory $\mathrm{CaMKII} \alpha+$ and putative inhibitory CaMKII $\alpha$ - cortical neurons after $24 \mathrm{~h}$ of exposure to methylglyoxal (Fig. 6). Our observations hint that the mechanistic underpinnings of AIS shortening by methylglyoxal in cortical neurons are different from homeostatic AIS shortening by $\mathrm{KCl}$ in hippocampal neurons.

If AIS shortening by methylglyoxal is not a mechanism of homeostatic plasticity, then how does neuronal function recover? AIS geometry is unlikely to be the sole determinant of network operation after methylglyoxal exposure. For example, homeostatic increase of synaptic input could lead to recovery of network activity despite AIS shortening. Indeed, methylglyoxal increases both 
evoked and spontaneous EPSPs in cockroach abdominal ganglia (Chambers et al., 1985), but whether it alters synaptic efficacy in mouse cortical neurons is unknown. The in vivo situation could be similar. Type 2 diabetic $\mathrm{db} / \mathrm{db}$ mice show AIS shortening (Yermakov et al., 2018, 2019), but there are also reports of altered synaptic plasticity (Li et al., 2002; Stranahan et al., 2008), decreased dendritic spine density (Stranahan et al., 2009; Chen et al., 2014), and progressive cortical atrophy (Ramos-Rodriguez et al., 2013). In addition, although not present in our in vitro model, elongation of the node of Ranvier after exposing myelinated CNS nerves to methylglyoxal ex vivo (Griggs et al., 2018) is similar to the elongated nodes present in older db/db mice (Yermakov et al., 2019). It is possible that the effects of methylglyoxal at the node of Ranvier in vivo could contribute to altered AIS geometry and network function. A better understanding of how concurrent changes at the AIS, node of Ranvier, and synapse affect network operation and cognitive function in neurological disease is needed. Finally, whether changes in AIS geometry and neuronal function are mechanistically linked remains an important unanswered question.

\section{AIS shortening may contribute to cognitive impairment}

Current and previous results suggest that methylglyoxal-evoked AIS shortening could be involved in the pathophysiology of diabetic brain complications. Methylglyoxal metabolism is disrupted in patients with diabetes (Sveen et al., 2013; Andersen et al., 2018) or cognitive deficits (Beeri et al., 2011; Srikanth et al., 2013; Cai et al., 2014). In animals, a single intracerebroventricular administration (Qi et al., 2017; Lissner et al., 2021) or repeated injection (Hansen et al., 2016; Szczepanik et al., 2020) of methylglyoxal induces cognitive impairment. These observations suggest that persistent elevation of methylglyoxal, such as in diabetes, could produce long-lasting changes in the operation of CNS networks in vivo. Importantly, methylglyoxal is increased in db/db mice (Bierhaus et al., 2012; Griggs et al., 2019) with cognitive impairment and shortened AIS in the prefrontal cortex and hippocampus (Yermakov et al., 2018, 2019), suggesting a connection between methylglyoxal, type 2 diabetes, AIS shortening, and cognitive impairment. Strengthening a mechanistic link between subtle changes in AIS geometry and CNS dysfunction, pharmacological treatment of nerve-injured mice simultaneously alleviated both AIS shortening and cognitive impairment (Shiers et al., 2018).

In conclusion, our results indicate that methylglyoxal is a key mediator of AIS shortening, depolarization, and network activity changes in cultured cortical neurons. We speculate that the persistent alteration of neuronal function and AIS length by chronic disruption of methylglyoxal metabolism in neurological disease might contribute to cognitive impairment. Further investigation of the mechanisms of methylglyoxal-evoked AIS shortening and perturbation of neuronal operation could have a beneficial impact on a wide variety of conditions associated with disrupted methylglyoxal metabolism such as type 2 diabetes, Alzheimer's disease, and aging.

\section{References}

Andersen ST, Witte DR, Dalsgaard EM, Andersen H, Nawroth P, Fleming T, Jensen TSM, Finnerup NB, Jensen TSM, Lauritzen T, Feldman EL, Callaghan BC, Charles M (2018) Risk factors for incident diabetic polyneuropathy in a cohort with screen-detected type 2 diabetes followed for 13 years: addition-Denmark. Diabetes Care 41:1068-1075.

Andersson DA, Gentry C, Light E, Vastani N, Vallortigara J, Bierhaus A, Fleming T, Bevan S (2013) Methylglyoxal evokes pain by stimulating TRPA1. PLoS One 8:e77986.

Atapour N, Rosa MGP (2017) Age-related plasticity of the axon initial segment of cortical pyramidal cells in marmoset monkeys. Neurobiol Aging 57:95-103.

Baalman KL, Cotton RJ, Rasband SN, Rasband MN (2013) Blast wave exposure impairs memory and decreases axon initial segment length. J Neurotrauma 30:741-751.

Beaudoin GMJJ, Lee SH, Singh D, Yuan Y, Ng YG, Reichardt LF, Arikkath J (2012) Culturing pyramidal neurons from the early postnatal mouse hippocampus and cortex. Nat Protoc 7:1741-1754.

Beeri MS, Moshier E, Schmeidler J, Godbold J, Uribarri J, Reddy S, Sano M, Grossman HT, Cai W, Vlassara H, Silverman JM (2011) Serum concentration of an inflammatory glycotoxin, methylglyoxal, is associated with increased cognitive decline in elderly individuals. Mech Ageing Dev 132:583-587.

Bélanger M, Yang J, Petit JM, Laroche T, Magistretti PJ, Allaman I (2011) Role of the glyoxalase system in astrocyte-mediated neuroprotection. J Neurosci 31:18338-18352.

Bender KJ, Trussell LO (2012) The physiology of the axon initial segment. Annu Rev Neurosci 35:249-265.

Bierhaus A, Fleming T, Stoyanov S, Leffler A, Babes A, Neacsu C, Sauer SK, Eberhardt M, Schnölzer M, Lasitschka F, Lasischka F, Neuhuber WL, Kichko TI, Konrade I, Elvert R, Mier W, Pirags V, Lukic IK, Morcos M, Dehmer T, et al. (2012) Methylglyoxal modification of Nav1.8 facilitates nociceptive neuron firing and causes hyperalgesia in diabetic neuropathy. Nat Med 18:926-933.

Biessels GJ, Despa F (2018) Cognitive decline and dementia in diabetes mellitus: mechanisms and clinical implications. Nat Rev Endocrinol 14:591-604.

Biessels GJ, Strachan MWJ, Visseren FLJ, Kappelle LJ, Whitmer RA (2014) Dementia and cognitive decline in type 2 diabetes and prediabetic stages: towards targeted interventions. Lancet Diabetes Endocrinol 2:246-255.

Buffington S, Rasband M (2011) The axon initial segment in nervous system disease and injury. Eur J Neurosci 34:1609-1619.

Cai W, Uribarri J, Zhu L, Chen X, Swamy S, Zhao Z, Grosjean F, Simonaro C, Kuchel GA, Schnaider-Beeri M, Woodward M, Striker GE, Vlassara H (2014) Oral glycotoxins are a modifiable cause of dementia and the metabolic syndrome in mice and humans. Proc Natl Acad Sci USA 111:4940-4945.

Chambers PL, Davies MG, Rowan MJ (1985) The effects of methylglyoxal on central synaptic transmission in the isolated nerve cord of the cockroach (Periplaneta americana L). Arch Toxicol Suppl 8:337-341.

Chand AN, Galliano E, Chesters RA, Grubb MS (2015) A distinct subtype of dopaminergic interneuron displays inverted structural plasticity at the axon initial segment. J Neurosci 35:1573-1590.

Charkhkar H, Meyyappan S, Matveeva E, Moll JR, McHail DG, Peixoto N, Cliff RO, Pancrazio JJ (2015) Amyloid beta modulation of neuronal network activity in vitro. Brain Res 1629:1-9.

Chen J, Liang L, Zhan L, Zhou Y, Zheng L, Sun X, Gong J, Sui H, Jiang $R$, Zhang $F$, Zhang $L$ (2014) ZiBuPiYin recipe protects $\mathrm{db} / \mathrm{db}$ mice from diabetes-associated cognitive decline through improving multiple pathological changes. PLoS One 9:e91680.

Clark KC, Sword BA, Dupree JL (2017) Oxidative stress induces disruption of the axon initial segment. ASN Neuro 9:1759091417745426.

de Arriba SG, Krügel U, Regenthal R, Vissiennon Z, Verdaguer E, Lewerenz A, García-Jordá E, Pallas M, Camins A, Münch G, Nieber K, Allgaier C (2006) Carbonyl stress and NMDA receptor 
activation contribute to methylglyoxal neurotoxicity. Free Radic Biol Med 40:779-790.

de Arriba SG, Stuchbury G, Yarin J, Burnell J, Loske C, Münch G (2007) Methylglyoxal impairs glucose metabolism and leads to energy depletion in neuronal cells-protection by carbonyl scavengers. Neurobiol Aging 28:1044-1050.

Ding Y, Chen T, Wang Q, Yuan Y, Hua T (2018) Axon initial segment plasticity accompanies enhanced excitation of visual cortical neurons in aged rats. Neuroreport 29:1537-1543.

Distler MG, Plant LD, Sokoloff G, Hawk AJ, Aneas I, Wuenschell GE, Termini J, Meredith SC, Nobrega MA, Palmer AA (2012) Glyoxalase 1 increases anxiety by reducing GABA A receptor agonist methylglyoxal. J Clin Invest 122:2306-2315.

Düll MM, Riegel K, Tappenbeck J, Ries V, Strupf M, Fleming T, Sauer SK, Namer B (2019) Methylglyoxal causes pain and hyperalgesia in human through C-fiber activation. Pain 160:2497-2507.

Eberhardt MJ, Filipovic MR, Leffler A, De La Roche J, Kistner K, Fischer MJ, Fleming T, Zimmermann K, Ivanovic-Burmazovic I, Nawroth PP, Bierhaus A, Reeh PW, Sauer SK (2012) Methylglyoxal activates nociceptors through transient receptor potential channel A1 (TRPA1): a possible mechanism of metabolic neuropathies. $J$ Biol Chem 287:28291-28306.

Evans MD, Sammons RP, Lebron S, Dumitrescu AS, Watkins TBK, Uebele VN, Renger JJ, Grubb MS (2013) Calcineurin signaling mediates activity-dependent relocation of the axon initial segment. $J$ Neurosci 33:6950-6963.

Evans MD, Dumitrescu AS, Kruijssen DLH, Taylor SE, Grubb MS (2015) Rapid modulation of axon initial segment length influences repetitive spike firing. Cell Rep 13:1233-1245.

Evans MD, Tufo C, Dumitrescu AS, Grubb MS (2017) Myosin II activity is required for structural plasticity at the axon initial segment. Eur J Neurosci 46:1751-1757.

Fong M, Newman JP, Potter SM, Wenner P (2015) Upward synaptic scaling is dependent on neurotransmission rather than spiking. Nat Commun 6:6339.

Fruscione F, Valente P, Sterlini B, Romei A, Baldassari S, Fadda M, Prestigio C, Giansante G, Sartorelli J, Rossi P, Rubio A, Gambardella A, Nieus T, Broccoli V, Fassio A, Baldelli P, Corradi A, Zara F, Benfenati F (2018) PRRT2 controls neuronal excitability by negatively modulating $\mathrm{Na}+$ channel 1.2/1.6 activity. Brain 141:1000-1016.

Galiano MR, Jha S, Ho TSY, Zhang C, Ogawa Y, Chang KJ, Stankewich MC, Mohler PJ, Rasband MN (2012) A distal axonal cytoskeleton forms an intra-axonal boundary that controls axon initial segment assembly. Cell 149:1125-1139.

Goethals S, Brette R (2020) Theoretical relation between axon initial segment geometry and excitability. Elife 9:834671.

Griggs RB, Yermakov LM, Drouet DE, Nguyen DVM, Susuki K (2018) Methylglyoxal disrupts paranodal axoglial junctions via calpain activation. ASN Neuro 10:1759091418766175.

Griggs RB, Santos DF, Laird DE, Doolen S, Donahue RR, Wessel CR, Fu W, Sinha GP, Wang P, Zhou J, Brings S, Fleming T, Nawroth PP, Susuki K, Taylor BK (2019) Methylglyoxal and a spinal TRPA1AC1-Epac cascade facilitate pain in the $\mathrm{db} / \mathrm{db}$ mouse model of type 2 diabetes. Neurobiol Dis 127:76-86.

Grubb MS, Burrone J (2010) Activity-dependent relocation of the axon initial segment fine-tunes neuronal excitability. Nature 465:1070-1074.

Guo Y, Su ZJ, Chen YK, Chai Z (2017) Brain-derived neurotrophic factor/neurotrophin 3 regulate axon initial segment location and affect neuronal excitability in cultured hippocampal neurons. J Neurochem 142:260-271.

Haddad M, Perrotte M, Ben Khedher MR, Demongin C, Lepage A, Fülöp T, Ramassamy C (2019) Methylglyoxal and glyoxal as potential peripheral markers for $\mathrm{MCl}$ diagnosis and their effects on the expression of neurotrophic, inflammatory and neurodegenerative factors in neurons and in neuronal derived-extracellular vesicles. Int J Mol Sci 20:4906.
Hales CM, Rolston JD, Potter SM (2010) How to culture, record and stimulate neuronal networks on micro-electrode arrays (MEAs). $J$ Vis Exp:1-7.

Hamada MS, Kole MHP (2015) Myelin loss and axonal ion channel adaptations associated with gray matter neuronal hyperexcitability. J Neurosci 35:7272-7286.

Hambsch B, Chen BG, Brenndörfer J, Meyer M, Avrabos C, MacCarrone G, Liu RH, Eder M, Turck CW, Landgraf R (2010) Methylglyoxal-mediated anxiolysis involves increased protein modification and elevated expression of glyoxalase 1 in the brain. J Neurochem 113:1240-1251.

Hansen F, Pandolfo P, Galland F, Torres FV, Dutra MF, Batassini C, Guerra MC, Leite MC, Gonçalves CA (2016) Methylglyoxal can mediate behavioral and neurochemical alterations in rat brain. Physiol Behav 164:93-101.

Harty RC, Kim TH, Thomas EA, Cardamone L, Jones NC, Petrou S, Wimmer VC (2013) Axon initial segment structural plasticity in animal models of genetic and acquired epilepsy. Epilepsy Res 105:272-279.

Hinman JD, Rasband MN, Carmichael ST (2013) Remodeling of the axon initial segment after focal cortical and white matter stroke. Stroke 44:182-189.

Huang CY, Rasband MN (2018) Axon initial segments: structure, function, and disease. Ann NY Acad Sci 1420:46-61.

Jamann N, Dannehl D, Lehmann N, Wagener R, Thielemann C, Schultz C, Staiger J, Kole MHP, Engelhardt M (2021) Sensory input drives rapid homeostatic scaling of the axon initial segment in mouse barrel cortex. Nat Commun 12:23.

Kim B, Sullivan KA, Backus C, Feldman EL (2011) Cortical neurons develop insulin resistance and blunted Akt signaling: a potential mechanism contributing to enhanced ischemic injury in diabetes. Antioxid Redox Signal 14:1829-1839.

Ko KW, Rasband MN, Meseguer V, Kramer RH, Golding NL (2016) Serotonin modulates spike probability in the axon initial segment through HCN channels. Nat Neurosci 19:1-7.

Kuhla B, Lüth HJ, Haferburg D, Boeck K, Arendt T, Münch G (2005) Methylglyoxal, glyoxal, and their detoxification in Alzheimer's disease. Ann NY Acad Sci 1043:211-216.

Kuhla B, Boeck K, Lüth HJ, Schmidt A, Weigle B, Schmitz M, Ogunlade V, Münch G, Arendt T (2006) Age-dependent changes of glyoxalase I expression in human brain. Neurobiol Aging 27:815822.

Kuhla B, Boeck K, Schmidt A, Ogunlade V, Arendt T, Münch G, Lüth $\mathrm{HJ}$ (2007) Age- and stage-dependent glyoxalase I expression and its activity in normal and Alzheimer's disease brains. Neurobiol Aging 28:29-41.

Kurz A, Rabbani N, Walter M, Bonin M, Thornalley P, Auburger G, Gispert S (2011) Alpha-synuclein deficiency leads to increased glyoxalase I expression and glycation stress. Cell Mol Life Sci 68:721-733.

Li XH, Xie JZ, Jiang X, Lv BL, Cheng XS, Du LL, Zhang JY, Wang JZ, Zhou XW (2012) Methylglyoxal induces tau hyperphosphorylation via promoting ages formation. NeuroMolecular Med 14:338-348.

Li XL, Aou S, Oomura Y, Hori N, Fukunaga K, Hori T (2002) Impairment of long-term potentiation and spatial memory in leptin receptor-deficient rodents. Neuroscience 113:607-615.

Lissner LJ, Rodrigues L, Wartchow KM, Borba E, Bobermin LD, Fontella FU, Hansen F, Quincozes-Santos A, Souza DOG, Gonçalves CA (2021) Short-term alterations in behavior and astroglial function after intracerebroventricular infusion of methylglyoxal in rats. Neurochem Res 46:183-196.

Liu CCC, Zhang XSS, Ruan YTT, Huang ZXX, Zhang SBB, Liu M, Luo HJJ, Wu SLL, Ma C (2017) Accumulation of methylglyoxal increases the advanced glycation end products levels in DRG and contributes to lumbar disc herniation-induced persistent pain. $J$ Neurophysiol 118:1321-1328.

Maeta K, Izawa S, Inoue Y (2005) Methylglyoxal, a metabolite derived from glycolysis, functions as a signal initiator of the high osmolarity glycerol-mitogen-activated protein kinase cascade and 
calcineurin/Crz1-mediated pathway in Saccharomyces cerevisiae. J Biol Chem 280:253-260.

Marin MA, Ziburkus J, Jankowsky J, Rasband MN (2016) Amyloid- $\beta$ plaques disrupt axon initial segments. Exp Neurol 281:93-98.

Neher E (1992) Correction for liquid junction potentials in patch clamp experiments. Methods Enzymol 207:123-131.

Nelson AD, Jenkins PM (2017) Axonal membranes and their domains: assembly and function of the axon initial segment and node of Ranvier. Front Cell Neurosci 11:136-117.

Nishimoto S, Koike S, Inoue N, Suzuki T, Ogasawara Y (2017) Activation of Nrf2 attenuates carbonyl stress induced by methylglyoxal in human neuroblastoma cells: increase in GSH levels is a critical event for the detoxi fi cation mechanism. Biochem Biophys Res Commun 483:1-6.

Percie du Sert N, Ahluwalia A, Alam S, Avey MT, Baker M, Browne WJ, Clark A, Cuthill IC, Dirnagl U, Emerson M, Garner P, Holgate ST, Howells DW, Hurst V, Karp NA, Lazic SE, Lidster K, MacCallum CJ, Macleod M, Pearl EJ, et al. (2020) Reporting animal research: explanation and elaboration for the ARRIVE guidelines 2.0. PLoS Biol 18:e3000411.

Potter SM, DeMarse TB (2001) A new approach to neural cell culture for long-term studies. J Neurosci Methods 110:17-24.

Prestigio C, Ferrante D, Valente P, Casagrande S, Albanesi E, Yanagawa Y, Benfenati F, Baldelli P (2019) Spike-related electrophysiological identification of cultured hippocampal excitatory and inhibitory neurons. Mol Neurobiol 56:6276-6292.

Qi LQ, Chen Z, Wang YP, Liu XY, Liu XH, Ke LF, Zheng Z, Lin XW, Zhou Y, Wu LJ, Bin Liu L (2017) Subcutaneous liraglutide ameliorates methylglyoxal-induced Alzheimer-like tau pathology and cognitive impairment by modulating tau hyperphosphorylation and glycogen synthase kinase-3 $\beta$. Am J Transl Res 9:247-260.

Rabbani N, Thornalley PJ (2012) Methylglyoxal, glyoxalase 1 and the dicarbonyl proteome. Amino Acids 42:1133-1142.

Rabbani N, Thornalley PJ (2014) Measurement of methylglyoxal by stable isotopic dilution analysis LC-MS/MS with corroborative prediction in physiological samples. Nat Protoc 9:1969-1979.

Radecki DZ, Johnson EL, Brown AK, Meshkin NT, Perrine SA, Gow A (2018) Corticohippocampal dysfunction in the OBiden mouse model of primary oligodendrogliopathy. Sci Rep 8:16116.

Radu BM, Dumitrescu DI, Mustaciosu CC, Radu M (2012) Dual effect of methylglyoxal on the intracellular $\mathrm{Ca}^{2+}$ signaling and neurite outgrowth in mouse sensory neurons. Cell Mol Neurobiol 32:1047-1057.

Ramos-Rodriguez JJ, Ortiz O, Jimenez-Palomares M, Kay KR, Berrocoso E, Murillo-Carretero MI, Perdomo G, Spires-Jones T, Cozar-Castellano I, Lechuga-Sancho AM, Garcia-Alloza M (2013) Differential central pathology and cognitive impairment in pre-diabetic and diabetic mice. Psychoneuroendocrinology 38:24622475.

Rasband MN (2010) The axon initial segment and the maintenance of neuronal polarity. Nat Rev Neurosci 11:552-562.

Sanders SS, Hernandez LM, Soh H, Karnam S, Walikonis RS, Tzingounis AV, Thomas GM (2020) The palmitoyl acyltransferase zdhhc14 controls kv1-family potassium channel clustering at the axon initial segment. Elife 9:e56058.

Schafer DP, Jha S, Liu F, Akella T, McCullough LD, Rasband MN (2009) Disruption of the axon initial segment cytoskeleton is a new mechanism for neuronal injury. J Neurosci 29:13242-13254.

Schalkwijk CG, Stehouwer CDA (2020) Methylglyoxal, a highly reactive dicarbonyl compound, in diabetes, its vascular complications, and other age-related diseases. Physiol Rev 100:407-461.

Shiers S, Pradhan G, Mwirigi J, Mejia G, Ahmad A, Kroener S, Price T (2018) Neuropathic pain creates an enduring prefrontal cortex dysfunction corrected by the type II diabetic drug metformin but not by gabapentin. J Neurosci 38:7337-7350.
Shimatani Y, Nodera H, Osaki Y, Banzrai C, Takayasu K, Endo S, Shibuta Y, Kaji R (2015) Upregulation of axonal HCN current by methylglyoxal: Potential association with diabetic polyneuropathy. Clin Neurophysiol 126:2226-2232.

Sohn PD, Huang CTL, Yan R, Fan L, Tracy TE, Camargo CM, Montgomery KM, Arhar T, Mok SA, Freilich R, Baik J, He M, Gong S, Roberson ED, Karch CM, Gestwicki JE, Xu K, Kosik KS, Gan L (2019) Pathogenic tau impairs axon initial segment plasticity and excitability homeostasis. Neuron 104:458-470.e5.

Sri S, Pegasiou CM, Cave CA, Hough K, Wood N, Gomez-Nicola D, Deinhardt K, Bannerman D, Perry VH, Vargas-Caballero M (2019) Emergence of synaptic and cognitive impairment in a matureonset APP mouse model of Alzheimer's disease. Acta Neuropathol Commun 7:25.

Srikanth V, Westcott B, Forbes J, Phan TG, Beare R, Venn A, Pearson S, Greenaway T, Parameswaran V, Münch G (2013) Methylglyoxal, cognitive function and cerebral atrophy in older people. J Gerontol A Biol Sci Med Sci 68:68-73.

Stranahan AM, Arumugam TV, Cutler RG, Lee K, Egan JM, Mattson MP (2008) Diabetes impairs hippocampal function through glucocorticoid-mediated effects on new and mature neurons. Nat Neurosci 11:309-317.

Stranahan AM, Lee K, Martin B, Maudsley S, Golden E, Cutler RG, Mattson MP (2009) Voluntary exercise and caloric restriction enhance hippocampal dendritic spine density and BDNF levels in diabetic mice. Hippocampus 19:951-961.

Sveen KA, Karimé B, Jørum E, Mellgren SI, Fagerland MW, Monnier VM, Dahl-Jørgensen K, Hanssen KF (2013) Small- and large-fiber neuropathy after 40 years of type 1 diabetes: associations with glycemic control and advanced protein glycation: the Oslo Study. Diabetes Care 36:3712-3717.

Szczepanik JC, de Almeida GRL, Cunha MP, Dafre AL (2020) Repeated methylglyoxal treatment depletes dopamine in the prefrontal cortex, and causes memory impairment and depressivelike behavior in mice. Neurochem Res 45:354-370.

Vascak M, Sun J, Baer M, Jacobs KM, Povlishock JT (2017) Mild traumatic brain injury evokes pyramidal neuron axon initial segment plasticity and diffuse presynaptic inhibitory terminal loss. Front Cell Neurosci 11:157.

Wagenaar DA, Pine J, Potter SM (2006) An extremely rich repertoire of bursting patterns during the development of cortical cultures. BMC Neurosci 7:11.

Wetzels S, Vanmierlo T, Scheijen JLJM, Van Horssen J, Amor S, Somers V, Schalkwijk CG, Hendriks JJA, Wouters K (2019) Methylglyoxal-derived advanced glycation endproducts accumulate in multiple sclerosis lesions. Front Immunol 10:1-9.

Yang Y, Ogawa Y, Hedstrom KL, Rasband MN (2007) $\beta$ IV spectrin is recruited to axon initial segments and nodes of Ranvier by ankyrinG. J Cell Biol 176:509-519.

Yau KW, van Beuningen SFB, Cunha-Ferreira I, Cloin BMC, van Battum EY, Will L, Schätzle P, Tas RP, van Krugten J, Katrukha EA, Jiang K, Wulf PS, Mikhaylova M, Harterink M, Pasterkamp RJ, Akhmanova A, Kapitein LC, Hoogenraad CC (2014) Microtubule minus-end binding protein CAMSAP2 controls axon specification and dendrite development. Neuron 82:1058-1073.

Yermakov LM, Drouet DE, Griggs RB, Elased KM, Susuki K (2018) Type 2 diabetes leads to axon initial segment shortening in $\mathrm{db} / \mathrm{db}$ mice. Front Cell Neurosci 12:146-113.

Yermakov LM, Griggs RB, Drouet DE, Sugimoto C, Williams MT, Vorhees CV, Susuki K (2019) Impairment of cognitive flexibility in type 2 diabetic db/db mice. Behav Brain Res 371:111978.

Zhang W, Bonadiman A, Ciorraga M, Benitez MJ, Garrido JJ (2019) P2Y1 purinergic receptor modulate axon initial segment initial development. Front Cell Neurosci 13:152-115. 